CRYSTALLOGRAPHIC COMMUNICATIONS

ISSN 2056-9890

\section{Crystal structure determination as part of an undergraduate laboratory experiment: $1^{\prime}, 3^{\prime}, 3^{\prime}$-trimethylspiro[chromene-2,2'-indoline] and $1^{\prime}, 3^{\prime}, 3^{\prime}$-trimethyl-4-[(E)-(1,3,3-trimethylindolin-2-yl- idene)methyl]spiro[chroman-2,2'-indoline]}

\author{
Joseph O. S. Beckett, Marilyn M. Olmstead, James C. Fettinger, David A. Gray, \\ Shuhei Manabe and Mark Mascal*
}

Received 11 August 2016
Accepted 10 October 2016

Edited by W. T. A. Harrison, University of Aberdeen, Scotland

Keywords: crystal structure; spiropyran; undergraduate teaching laboratory.

CCDC references: $1509185 ; 1509184$

Supporting information: this article has supporting information at journals.iucr.org/e

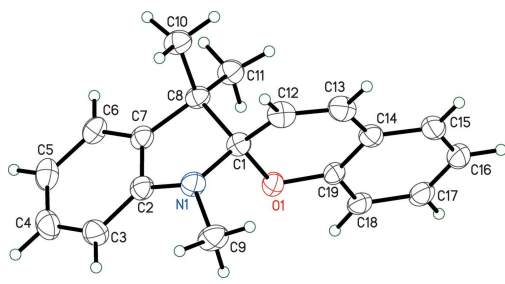

OPEN $\odot$ ACCESS
Department of Chemistry, University of California, Davis, One Shields Avenue, Davis, CA 95616, USA. *Correspondence e-mail:mjmascal@ucdavis.edu

The crystal structures of the title compounds, $\mathrm{C}_{19} \mathrm{H}_{19} \mathrm{NO}$ and $\mathrm{C}_{31} \mathrm{H}_{34} \mathrm{~N}_{2} \mathrm{O}$, were determined as part of an experiment in an undergraduate teaching laboratory $1^{\prime}, 3^{\prime}, 3^{\prime}$-Trimethylspiro[chromene-2,2'-indoline] is both a photoswitch and thermochromic molecule. Students synthesized it and a bis-indoline adduct and compared the crystallographically determined structures to computed gasphase models.

\section{Chemical context}

In an ever evolving pursuit to improve the educational experience in undergraduate organic chemistry laboratory courses, we introduced an experiment in which students prepare a 'functional molecule,' in this case spiropyran $\mathbf{1}$. Compounds such as $\mathbf{1}$ are broadly characterized as 'responsive,' due to their ability to be actuated by a range of stimuli, including light, heat, metal ions, $\mathrm{pH}$, mechanical force, and changes in solvent polarity (Klajn, 2014). An advantage of the spiropyran system over other photochromic/thermochromic materials is the strongly differentiated electronic forms between which equilibrium is shifted. The closed-ring isomer of $\mathbf{1}$ comprises an indoline and a chromene ring bound together at a spiro junction, while the open-ring form is a zwitterionic merocyanine $\mathbf{1 a}$ (Scheme 1).

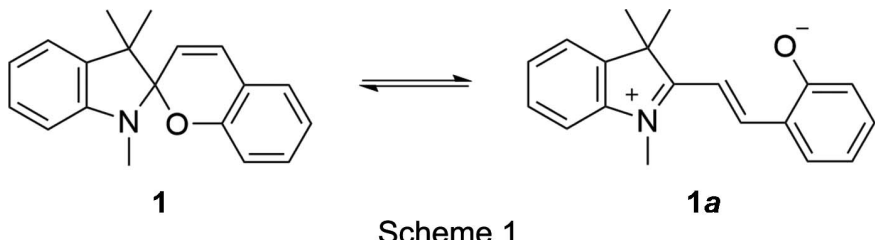

Although a variety of substituted spiropyran derivatives are known in the literature, for simplicity, we elected to focus on the unsubstituted parent compound, which is colorless in its closed form and red in its open form. The molecule was synthesized in a single step by condensation of 1,3,3-trimethyl2-methyleneindoline with salicylaldehyde (Koelsch \& Workman, 1952). The methyleneindoline nucleophile can also react a second time with $\mathbf{1}$ to give the bis adduct $\mathbf{2}$ as a side product (Scheme 2). that demonstrates the relationship between molecular structure and function. 
Since this experiment was oriented around the functional attributes of $\mathbf{1}$, it presented an ideal opportunity to introduce structural characterization methods into the laboratory course, since the function of $\mathbf{1}$ is directly linked to its structure. Students first model the two forms of $\mathbf{1}$ using both molecular mechanics and semi-empirical quantum mechanical methods. These calculations indicate that the spiropyran form of $\mathbf{1}$ is more stable than the open form 1a. They then grow crystals of 1 by slow evaporation from acetone, resulting in most cases in large (up to $10 \mathrm{~mm} \times 10 \mathrm{~mm}$ ), thin pink plates. Although the students do not themselves determine the X-ray crystal structure, crystallographic characterization of $\mathbf{1}$ has allowed students to compare gas-phase models with condensed-state empirical data.

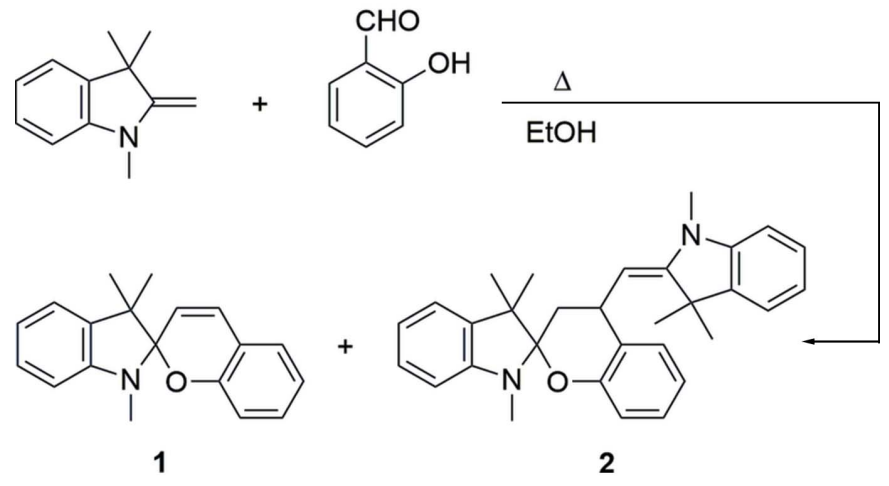

Scheme 2

\section{Structural commentary}

Crystals of the parent spiropyran, $1^{\prime}, 3^{\prime}, 3^{\prime}$-trimethylspiro[chromene-2,2'-indoline] $\mathbf{1}$, are colorless at low temperature $(90 \mathrm{~K})$. Fig. 1 depicts the low-temperature crystal structure. There is one molecule in the asymmetric unit. The central $s p^{3}$ carbon atom, $\mathrm{C} 1$, has a tetrahedral geometry. The dihedral angle between $\mathrm{O} 1 / \mathrm{C} 1 / \mathrm{C} 12$ and $\mathrm{N} 1 / \mathrm{C} 1 / \mathrm{C} 8$ is $89.33(12)^{\circ}$. The $\mathrm{C} 12-\mathrm{C} 13$ bond is a double bond with a length of 1.330 (3) $\AA$. The substituted spiropyran, 1',3',3'-trimethyl-4-[(E)-(1,3,3-trimethylindolin-2-ylidene)methyl]spiro[chroman-2,2'-indoline] $\mathbf{2}$, is also colorless at low temperature. It differs from $\mathbf{1}$ by

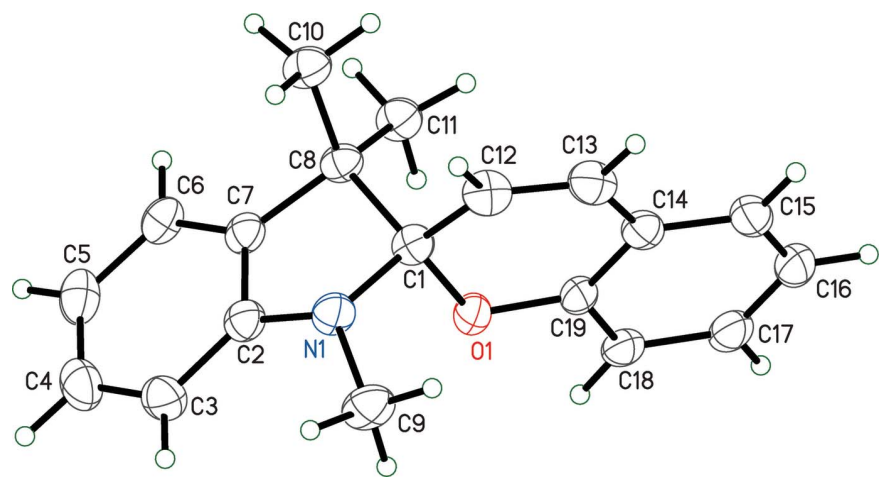

Figure 1

The molecular structure of $\mathbf{1}$. Displacement parameters are shown at the $50 \%$ probability level. virtue of substitution at $\mathrm{C} 13$ with a methyleneindoline group (Fig. 2). Consequently, $\mathrm{C} 12$ and $\mathrm{C} 13$ are now singly bonded, with a distance of 1.5367 (14) $\AA$. The central carbon atom remains tetrahedral with the value of the dihedral angle at 89.69 (5), comparable to 1 . The atoms $\mathrm{C} 1$ and $\mathrm{C} 13$ have the same chirality, either $R R$ or $S S$.

Differences between molecular mechanics force field MM2 calculations and the semi-empirical quantum mechanical methods PM6 and PDDG versus experimental X-ray values for selected bond lengths and angles can be seen in Table 1. A clear trend in the data is reflected in the fact that thermal motion in low-temperature X-ray diffraction experiments tends to lead to an apparent bond shortening. Considering only those distances not involving phenyl carbon atoms, the data indicate that MM2 shows the poorest mean agreement with X-ray in bond lengths $( \pm 0.043 \AA)$, while PDDG $( \pm 0.021 \AA)$ and PM6 $( \pm 0.017 \AA)$ perform better. The most serious modeling failure was in the MM2 N1 $-\mathrm{C} 2$ bond which, at $1.270 \AA$, was interpreted by molecular mechanics to be a double bond, but which was clearly a single bond in the X-ray structure at 1.405 (2) $\AA$. As a consequence, the sum of the angles at $\mathrm{N} 1$ was $360^{\circ}$ in the MM2 calculation, whereas the experimental value was $348.36^{\circ}$. PM6 and PDDG again performed better here, with sums of 345.4 and $344.5^{\circ}$, respectively. The dihedral angle between the $01 / \mathrm{C} 1 / \mathrm{C} 2$ plane and the $\mathrm{N} 1 / \mathrm{C} 1 / \mathrm{C} 8$ plane was $89.3^{\circ}$ for $\mathrm{X}$-ray, compared to $92.7^{\circ}$ for MM2, $91.3^{\circ}$ for PM6 and $91.4^{\circ}$ for PDDG. Bond angle

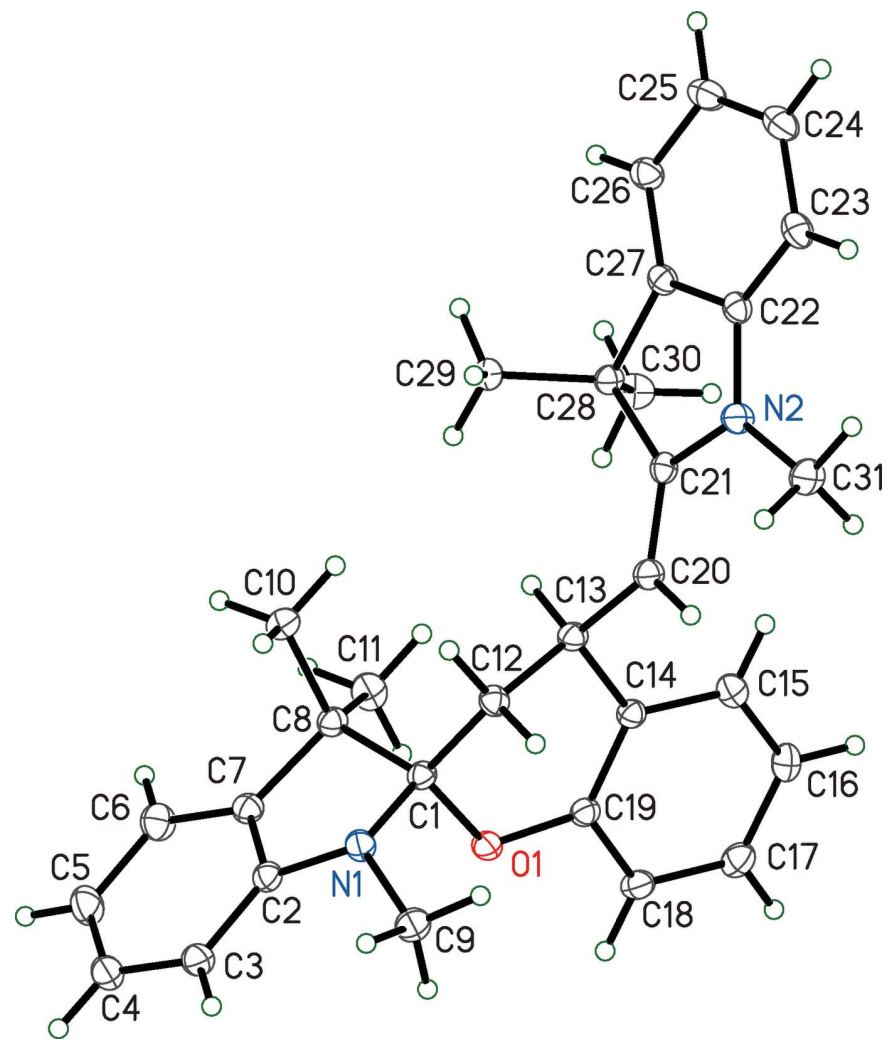

Figure 2

The molecular structure of $\mathbf{2}$. Displacement parameters are shown at the $50 \%$ probability level. 
Table 1

Comparison of modeled (MM2, PDDG, PM6) bond lengths, angles, and dihedral angles $\left(\AA \mathrm{\circ}^{\circ}\right)$ with X-ray crystallographic data.

\begin{tabular}{|c|c|c|c|c|c|c|c|}
\hline & X-ray & MM2 & $\Delta$ & PDDG & $\Delta$ & PM6 & $\Delta$ \\
\hline $\mathrm{C} 1-\mathrm{O} 1$ & 1.471 & 1.415 & 0.056 & 1.423 & 0.048 & 1.484 & -0.013 \\
\hline $\mathrm{C} 1-\mathrm{N} 1$ & 1.447 & 1.488 & -0.041 & 1.515 & -0.068 & 1.493 & -0.046 \\
\hline $\mathrm{C} 1-\mathrm{C} 8$ & 1.580 & 1.588 & -0.008 & 1.589 & -0.009 & 1.599 & -0.019 \\
\hline $\mathrm{N} 1-\mathrm{C} 2$ & 1.405 & 1.270 & 0.135 & 1.428 & -0.023 & 1.430 & -0.025 \\
\hline $\mathrm{N} 1-\mathrm{C} 9$ & 1.457 & 1.475 & -0.018 & 1.468 & -0.011 & 1.481 & -0.024 \\
\hline $\mathrm{C} 12-\mathrm{C} 13$ & 1.330 & 1.338 & -0.008 & 1.340 & -0.010 & 1.340 & -0.010 \\
\hline $\mathrm{C} 13-\mathrm{C} 14$ & 1.453 & 1.343 & 0.110 & 1.448 & 0.005 & 1.455 & -0.002 \\
\hline |mean| & & & 0.043 & & 0.021 & & 0.017 \\
\hline Dihedral angle $\mathrm{O} 1 / \mathrm{C} 1 / \mathrm{C} 12$ and $\mathrm{N} 1 / \mathrm{C} 1 / \mathrm{C} 8$ & 89.33 & 92.7 & -3.370 & 91.4 & -2.070 & 91.3 & -1.970 \\
\hline Sum of angles at $\mathrm{N} 1$ & 348.36 & 360.0 & -11.640 & 345.4 & 2.960 & 344.5 & 3.860 \\
\hline $\mathrm{C} 1-\mathrm{O} 1-\mathrm{C} 19$ & 121.03 & 119.1 & 1.93 & 118.4 & 2.63 & 121.3 & -0.27 \\
\hline $\mathrm{O} 1-\mathrm{C} 1-\mathrm{C} 12$ & 111.35 & 111.3 & 0.05 & 115.4 & -4.05 & 113.7 & -2.35 \\
\hline $\mathrm{C} 8-\mathrm{C} 1-\mathrm{C} 12$ & 114.70 & 114.0 & 0.70 & 112.3 & 2.40 & 116.6 & -1.90 \\
\hline |mean| & & & 2.08 & & 2.63 & & 1.99 \\
\hline
\end{tabular}

deviations ranged from 0 to $5^{\circ}$ and averaged $c a 2^{\circ}$ for all three methods. Interestingly, if the two angles in poor agreement around $\mathrm{C} 1$ are discarded, MM2 actually performs somewhat better than the semi-empirical models for angle data. If all data in Table 1 are taken into account, PM6 is seen to outperform both PDDG and MM2.

\section{Supramolecular features}

The KPI of $\mathbf{1}$ is $68.7 \%$ and that of $\mathbf{2}$ is $69.6 \%$ (van der Sluis \& Spek, 1990). Neither structure has significant directional intermolecular interactions.

\section{Database survey}

There are 67 structures in the CSD (Groom et al., 2016) with the basic skeleton of compound $\mathbf{1}$. All of these are substituted in one way or another. There are no unusual differences among these structures. Since the $\mathrm{C} 1-\mathrm{O} 1$ bond is broken in the transformation to the merocyanine form, it is of interest to examine this bond length. Of the 82 hits with similar geometry, the mean $\mathrm{C}-\mathrm{O}$ distance in the CSD is $1.479(15)^{\circ}$. For $\mathbf{1}$, this distance is $1.4708(19) \AA$. For 2 , the same distance is 1.4648 (12) A. There are five structures in the CSD that involve further methyleneindoline substitution, similar to 2 . In all cases, the structures are racemic and the chirality is either $R R$ or $S S$. Two of the deposits (NESZOC and NESZOC01; Ashraf et al., 2012) describe the results from two different crystals, two different radiations $(\mathrm{Cu} K \alpha$ and $\mathrm{Mo} K \alpha)$, and two different temperatures (153 and $113 \mathrm{~K}$ ), respectively. Structurally, there is no significant difference between them, but the higher temperature crystal is described as a red prism while the lower temperature crystal is a pink plate. This feature was not discussed, but it raises the possibility of a merocyanine impurity arising due to the thermochromic effect.

\section{Synthesis and crystallization}

A solution of 1,3,3-trimethyl-2-methyleneindoline (3.37 g, $19.5 \mathrm{mmol})$ and salicylaldehyde $(2.53 \mathrm{~g}, 20.7 \mathrm{mmol})$ in absolute ethanol $(15 \mathrm{~mL})$ was heated at reflux with stirring for $1 \mathrm{~h}$. A white precipitate was filtered from the hot solution and washed with cold absolute ethanol. The solid was recrystallized from acetone to give $1^{\prime}, 3^{\prime}, 3^{\prime}$-trimethyl-4- $[(E)-(1,3,3-$ trimethylindolin-2-ylidene)methyl]spiro[chroman-2,2' -indoline] $2(0.49 \mathrm{~g}, 11 \%)$, m.p. 474-477 K. The filtrate/wash was then evaporated and the residue was recrystallized from $90 \%$ ethanol to give $1^{\prime}, 3^{\prime}, 3^{\prime}$-trimethylspiro[chromene-2,2'-indoline] $\mathbf{1}(2.58 \mathrm{~g}, 48 \%$ ), m.p. 366-368 K. Crystals of $\mathbf{1}$ and $\mathbf{2}$ suitable for $\mathrm{X}$-ray diffraction were obtained by slow evaporation from acetone solutions.

\section{Refinement}

Crystal data, data collection and structure refinement details are summarized in Table 2. The hydrogen atoms bonded to carbon were located by geometry and refined using a riding model. Distances were fixed at $0.95 \AA$ for $\mathrm{C}-\mathrm{H}$ bonds in phenyl rings and $0.98 \AA$ in methyl groups. In structure 2 , primary $\mathrm{C}-\mathrm{H}$ bonds were assigned $\mathrm{C}-\mathrm{H}$ distances of $1.00 \AA$ while secondary $\mathrm{C}-\mathrm{H}$ distances were given values of $0.99 \AA$. The $U_{\text {iso }}(\mathrm{H})$ parameters were set equal to $1.5 U_{\text {eq }}$ for the methyl groups and to $1.2 U_{\mathrm{eq}}$ of the parent carbon for all others. 
Table 2

Experimental details.

1

Crystal data

Chemical formula

$M_{\mathrm{r}}$

Crystal system, space group

Temperature (K)

$a, b, c(\AA)$

$\beta\left({ }^{\circ}\right)$

$V\left(\AA^{3}\right)$

$Z$

Radiation type

$\mu\left(\mathrm{mm}^{-1}\right)$

Crystal size (mm)

Data collection

Diffractometer

Absorption correction

$T_{\min }, T_{\max }$

No. of measured, independent and observed $[I>2 \sigma(I)]$ reflections

$R_{\text {int }}$

$(\sin \theta / \lambda)_{\max }\left(\AA^{-1}\right)$

Refinement

$R\left[F^{2}>2 \sigma\left(F^{2}\right)\right], w R\left(F^{2}\right), S$

No. of reflections

No. of parameters

$\mathrm{H}$-atom treatment

$\Delta \rho_{\max }, \Delta \rho_{\min }\left({\left.\mathrm{e} \AA^{-3}\right)}^{-3}\right.$

2

\begin{tabular}{|c|c|}
\hline $\mathrm{C}_{19} \mathrm{H}_{19} \mathrm{NO}$ & $\mathrm{C}_{31} \mathrm{H}_{34} \mathrm{~N}_{2} \mathrm{O}$ \\
\hline 277.35 & 450.60 \\
\hline Monoclinic, $P 2_{1} / c$ & Monoclinic, $P 2_{1} / c$ \\
\hline 90 & 90 \\
\hline $11.530(7), 10.938(6), 13.013(7)$ & $\begin{array}{l}14.1774(11), 11.6019(9), \\
\quad 16.2847(17)\end{array}$ \\
\hline $115.614(7)$ & $115.6129(12)$ \\
\hline $1479.9(15)$ & $2415.4(4)$ \\
\hline 4 & 4 \\
\hline Мo $K \alpha$ & Мо $K \alpha$ \\
\hline 0.08 & 0.07 \\
\hline $0.52 \times 0.36 \times 0.35$ & $0.48 \times 0.26 \times 0.08$ \\
\hline Bruker SMART 1000 & Bruker DUO \\
\hline $\begin{array}{l}\text { Multi-scan (SADABS; Bruker, } \\
\text { 2014) }\end{array}$ & $\begin{array}{l}\text { Multi-scan (SADABS; Bruker, } \\
\text { 2014) }\end{array}$ \\
\hline $0.811,0.983$ & $0.713,0.746$ \\
\hline $12543,3358,2672$ & $39237,7680,6549$ \\
\hline 0.029 & 0.026 \\
\hline 0.650 & 0.725 \\
\hline $0.050,0.139,1.05$ & $0.046,0.124,1.03$ \\
\hline 3358 & 7680 \\
\hline 193 & 313 \\
\hline $\mathrm{H}$-atom parameters constrained & $\mathrm{H}$-atom parameters constrained \\
\hline $0.23,-0.23$ & $0.61,-0.22$ \\
\hline
\end{tabular}

$\mathrm{C}_{19} \mathrm{H}_{19} \mathrm{NO}$

277.35

90

$115.614(7)$

$1479.9(15)$

Mo $K \alpha$

0.08

$0.52 \times 0.36 \times 0.35$

Bruker SMART 1000

Multi-scan (SADABS; Bruker, 2014)

$0.811,0.983$

Computer programs: SMART (Bruker, 2002), SAINT (Bruker, 2013, 2014), APEX2 (Bruker, 2014), SHELXTL (Sheldrick, 2008), SHELXT (Sheldrick, 2015a) and SHELXL2014 (Sheldrick, 2015b).

\section{Acknowledgements}

We thank the National Science Foundation (Grant 0840444) for the Dual source X-ray diffractometer.

\section{References}

Ashraf, M., Gainsford, G. J. \& Kay, A. J. (2012). Aust. J. Chem. 65, 779-784.

Bruker (2002). SMART. Bruker AXS Inc., Madison, Wisconsin, USA.
Bruker (2013). SAINT. Bruker AXS Inc., Madison, Wisconsin, USA. Bruker (2014). APEX2 and $S A D A B S$. Bruker AXS Inc., Madison, Wisconsin, USA.

Groom, C. R., Bruno, I. J., Lightfoot, M. P. \& Ward, S. C. (2016). Acta Cryst. B72, 171-179.

Klajn, R. (2014). Chem. Soc. Rev. 43, 148-184.

Koelsch, C. F. \& Workman, W. R. (1952). J. Am. Chem. Soc. 74, 62886289.

Sheldrick, G. M. (2008). Acta Cryst. A64, 112-122.

Sheldrick, G. M. (2015a). Acta Cryst. A71, 3-8.

Sheldrick, G. M. (2015b). Acta Cryst. C71, 3-8.

Sluis, P. van der \& Spek, A. L. (1990). Acta Cryst. A46, 194-201. 


\section{supporting information}

Acta Cryst. (2016). E72, 1659-1662 [https://doi.org/10.1107/S2056989016016042]

Crystal structure determination as part of an undergraduate laboratory experiment: $1^{\prime}, 3^{\prime}, 3^{\prime}$-trimethylspiro[chromene-2,2'-indoline] and 1', $3^{\prime}, 3^{\prime}$-trimethyl-4-[(E)-(1,3,3-trimethylindolin-2-ylidene)methyl]spiro[chroman-2,2'indoline]

Joseph O. S. Beckett, Marilyn M. Olmstead, James C. Fettinger, David A. Gray, Shuhei Manabe and Mark Mascal

Computing details

Data collection: SMART (Bruker, 2002) for (1); APEX2 (Bruker, 2014) for (2). Cell refinement: SAINT (Bruker, 2013) for (1); SAINT (Bruker, 2014) for (2). Data reduction: SAINT (Bruker, 2013) for (1); SAINT (Bruker, 2014) for (2). For both compounds, program(s) used to solve structure: SHELXT (Sheldrick, 2015a); program(s) used to refine structure:

SHELXL2014 (Sheldrick, 2015b); molecular graphics: SHELXTL (Sheldrick, 2008); software used to prepare material for publication: SHELXL2014 (Sheldrick, 2015b).

(1) 1',3',3'-Trimethylspiro[chromene-2,2'-indoline]

Crystal data

$\mathrm{C}_{19} \mathrm{H}_{19} \mathrm{NO}$

$M_{r}=277.35$

Monoclinic, $P 2_{1} / c$

$a=11.530(7) \AA$

$b=10.938(6) \AA$

$c=13.013(7) \AA$

$\beta=115.614(7)^{\circ}$

$V=1479.9(15) \AA^{3}$

$Z=4$

$F(000)=592$

$D_{\mathrm{x}}=1.245 \mathrm{Mg} \mathrm{m}^{-3}$

Mo $K \alpha$ radiation, $\lambda=0.71073 \AA$

Cell parameters from 9931 reflections

$\theta=2.6-27.4^{\circ}$

$\mu=0.08 \mathrm{~mm}^{-1}$

$T=90 \mathrm{~K}$

Block, colorless

$0.52 \times 0.36 \times 0.35 \mathrm{~mm}$

Data collection

Bruker SMART 1000

diffractometer

Radiation source: fine-focus sealed tube

Detector resolution: 8.3 pixels $\mathrm{mm}^{-1}$

$\omega$ scans

Absorption correction: multi-scan

(SADABS; Bruker, 2014)

$T_{\min }=0.811, T_{\max }=0.983$

12543 measured reflections

3358 independent reflections

2672 reflections with $I>2 \sigma(I)$

$R_{\text {int }}=0.029$

$\theta_{\text {max }}=27.5^{\circ}, \theta_{\min }=2.0^{\circ}$

$h=-14 \rightarrow 14$

$k=-14 \rightarrow 14$

$l=-16 \rightarrow 16$ 


\section{Refinement}

Refinement on $F^{2}$

Least-squares matrix: full

$R\left[F^{2}>2 \sigma\left(F^{2}\right)\right]=0.050$

$w R\left(F^{2}\right)=0.139$

$S=1.05$

3358 reflections

193 parameters

0 restraints

Primary atom site location: dual
Secondary atom site location: difference Fourier map

Hydrogen site location: inferred from neighbouring sites

$\mathrm{H}$-atom parameters constrained

$w=1 /\left[\sigma^{2}\left(F_{\mathrm{o}}^{2}\right)+(0.0554 P)^{2}+1.046 P\right]$ where $P=\left(F_{\mathrm{o}}{ }^{2}+2 F_{\mathrm{c}}{ }^{2}\right) / 3$

$(\Delta / \sigma)_{\max }<0.001$

$\Delta \rho_{\max }=0.23 \mathrm{e} \AA^{-3}$

$\Delta \rho_{\min }=-0.23$ e $\AA^{-3}$

Special details

Geometry. All esds (except the esd in the dihedral angle between two 1.s. planes) are estimated using the full covariance matrix. The cell esds are taken into account individually in the estimation of esds in distances, angles and torsion angles; correlations between esds in cell parameters are only used when they are defined by crystal symmetry. An approximate (isotropic) treatment of cell esds is used for estimating esds involving l.s. planes.

Fractional atomic coordinates and isotropic or equivalent isotropic displacement parameters $\left(\AA^{2}\right)$

\begin{tabular}{lllll}
\hline & $x$ & $y$ & $z$ & $U_{\text {iso }} / U_{\text {eq }}$ \\
\hline O1 & $0.80339(11)$ & $0.34663(10)$ & $0.35758(9)$ & $0.0279(3)$ \\
N1 & $0.80594(13)$ & $0.55031(13)$ & $0.30705(12)$ & $0.0296(3)$ \\
C1 & $0.73425(15)$ & $0.43762(14)$ & $0.26886(13)$ & $0.0271(3)$ \\
C2 & $0.76732(15)$ & $0.60633(14)$ & $0.38435(14)$ & $0.0284(3)$ \\
C3 & $0.83102(17)$ & $0.69436(16)$ & $0.46624(15)$ & $0.0362(4)$ \\
H3 & 0.9137 & 0.7230 & 0.4784 & $0.043^{*}$ \\
C4 & $0.76958(19)$ & $0.73941(16)$ & $0.53015(16)$ & $0.0392(4)$ \\
H4 & 0.8110 & 0.8002 & 0.5863 & $0.047^{*}$ \\
C5 & $0.64926(19)$ & $0.69733(16)$ & $0.51348(15)$ & $0.0373(4)$ \\
H5 & 0.6089 & 0.7298 & 0.5576 & $0.045^{*}$ \\
C6 & $0.58729(16)$ & $0.60716(15)$ & $0.43184(14)$ & $0.0310(4)$ \\
H6 & 0.5050 & 0.5777 & 0.4203 & $0.037^{*}$ \\
C7 & $0.64723(15)$ & $0.56145(14)$ & $0.36826(13)$ & $0.0267(3)$ \\
C8 & $0.60256(14)$ & $0.46823(14)$ & $0.27321(13)$ & $0.0261(3)$ \\
C9 & $0.93940(16)$ & $0.55554(18)$ & $0.32437(17)$ & $0.0386(4)$ \\
H9A & 0.9487 & 0.5105 & 0.2633 & $0.058^{*}$ \\
H9B & 0.9948 & 0.5187 & 0.3981 & $0.058^{*}$ \\
H9C & 0.9645 & 0.6410 & 0.3234 & $0.058^{*}$ \\
C10 & $0.50907(16)$ & $0.52895(16)$ & $0.16130(14)$ & $0.0329(4)$ \\
H10A & 0.4821 & 0.4690 & 0.0993 & $0.049^{*}$ \\
H10B & 0.5521 & 0.5976 & 0.1437 & $0.049^{*}$ \\
H10C & 0.4335 & 0.5590 & 0.1696 & $0.049^{*}$ \\
C11 & $0.53771(16)$ & $0.35560(15)$ & $0.29460(15)$ & $0.0312(4)$ \\
H11A & 0.5220 & 0.2952 & 0.2344 & $0.047^{*}$ \\
H11B & 0.4557 & 0.3794 & 0.2945 & $0.047^{*}$ \\
H11C & 0.5938 & 0.3199 & 0.3687 & $0.047^{*}$ \\
C12 & $0.72190(17)$ & $0.39690(17)$ & $0.15482(14)$ & $0.0336(4)$ \\
H12 & 0.7053 & 0.4565 & 0.0970 & $0.040^{*}$ \\
& & & &
\end{tabular}


supporting information

\begin{tabular}{lllll} 
C13 & $0.73330(16)$ & $0.28041(17)$ & $0.13139(14)$ & $0.0339(4)$ \\
H13 & 0.7175 & 0.2579 & 0.0560 & $0.041^{*}$ \\
C14 & $0.76943(15)$ & $0.18690(15)$ & $0.21904(14)$ & $0.0290(3)$ \\
C15 & $0.77489(16)$ & $0.06223(16)$ & $0.19799(16)$ & $0.0343(4)$ \\
H15 & 0.7521 & 0.0349 & 0.1224 & $0.041^{*}$ \\
C16 & $0.81308(16)$ & $-0.02187(16)$ & $0.28582(17)$ & $0.0372(4)$ \\
H16 & 0.8147 & -0.1066 & 0.2704 & $0.045^{*}$ \\
C17 & $0.84899(15)$ & $0.01812(16)$ & $0.39650(16)$ & $0.0344(4)$ \\
H17 & 0.8755 & -0.0395 & 0.4570 & $0.041^{*}$ \\
C18 & $0.84650(14)$ & $0.14200(15)$ & $0.41974(14)$ & $0.0290(3)$ \\
H18 & 0.8735 & 0.1692 & 0.4960 & $0.035^{*}$ \\
C19 & $0.80426(14)$ & $0.22559(14)$ & $0.33084(13)$ & $0.0261(3)$ \\
\hline
\end{tabular}

Atomic displacement parameters $\left(\AA^{2}\right)$

\begin{tabular}{lllllll}
\hline & $U^{11}$ & $U^{22}$ & $U^{33}$ & $U^{12}$ & $U^{13}$ & $U^{23}$ \\
\hline O1 & $0.0296(6)$ & $0.0243(5)$ & $0.0275(6)$ & $0.0041(4)$ & $0.0103(5)$ & $0.0002(4)$ \\
N1 & $0.0238(7)$ & $0.0291(7)$ & $0.0385(8)$ & $-0.0025(5)$ & $0.0159(6)$ & $0.0002(6)$ \\
C1 & $0.0259(7)$ & $0.0271(8)$ & $0.0285(8)$ & $0.0003(6)$ & $0.0119(6)$ & $0.0029(6)$ \\
C2 & $0.0269(8)$ & $0.0243(7)$ & $0.0331(8)$ & $0.0008(6)$ & $0.0122(7)$ & $0.0031(6)$ \\
C3 & $0.0346(9)$ & $0.0276(8)$ & $0.0402(9)$ & $-0.0035(7)$ & $0.0104(7)$ & $-0.0001(7)$ \\
C4 & $0.0511(11)$ & $0.0254(8)$ & $0.0361(9)$ & $0.0006(7)$ & $0.0142(8)$ & $-0.0005(7)$ \\
C5 & $0.0509(11)$ & $0.0284(8)$ & $0.0362(9)$ & $0.0089(8)$ & $0.0223(8)$ & $0.0036(7)$ \\
C6 & $0.0318(8)$ & $0.0278(8)$ & $0.0360(8)$ & $0.0057(6)$ & $0.0172(7)$ & $0.0065(7)$ \\
C7 & $0.0266(7)$ & $0.0234(7)$ & $0.0292(8)$ & $0.0028(6)$ & $0.0112(6)$ & $0.0043(6)$ \\
C8 & $0.0230(7)$ & $0.0271(8)$ & $0.0279(8)$ & $0.0000(6)$ & $0.0106(6)$ & $0.0037(6)$ \\
C9 & $0.0254(8)$ & $0.0435(10)$ & $0.0490(10)$ & $-0.0023(7)$ & $0.0180(8)$ & $0.0036(8)$ \\
C10 & $0.0282(8)$ & $0.0340(9)$ & $0.0333(9)$ & $0.0012(7)$ & $0.0102(7)$ & $0.0059(7)$ \\
C11 & $0.0280(8)$ & $0.0295(8)$ & $0.0363(9)$ & $-0.0024(6)$ & $0.0143(7)$ & $0.0036(7)$ \\
C12 & $0.0338(9)$ & $0.0399(9)$ & $0.0291(8)$ & $-0.0008(7)$ & $0.0154(7)$ & $0.0027(7)$ \\
C13 & $0.0315(8)$ & $0.0438(10)$ & $0.0287(8)$ & $-0.0027(7)$ & $0.0151(7)$ & $-0.0041(7)$ \\
C14 & $0.0218(7)$ & $0.0350(9)$ & $0.0317(8)$ & $-0.0017(6)$ & $0.0130(6)$ & $-0.0055(7)$ \\
C15 & $0.0244(8)$ & $0.0383(9)$ & $0.0405(9)$ & $-0.0031(7)$ & $0.0144(7)$ & $-0.0123(7)$ \\
C16 & $0.0259(8)$ & $0.0298(9)$ & $0.0528(11)$ & $-0.0002(7)$ & $0.0143(8)$ & $-0.0079(8)$ \\
C17 & $0.0226(8)$ & $0.0298(8)$ & $0.0474(10)$ & $0.0014(6)$ & $0.0118(7)$ & $0.0021(7)$ \\
C18 & $0.0204(7)$ & $0.0317(8)$ & $0.0330(8)$ & $0.0017(6)$ & $0.0096(6)$ & $0.0007(7)$ \\
C19 & $0.0196(7)$ & $0.0268(8)$ & $0.0330(8)$ & $0.0007(6)$ & $0.0125(6)$ & $-0.0037(6)$ \\
& & & & & &
\end{tabular}

Geometric parameters $\left(\AA,{ }^{\circ}\right)$

\begin{tabular}{llll}
\hline $\mathrm{O} 1-\mathrm{C} 19$ & $1.370(2)$ & $\mathrm{C} 9-\mathrm{H} 9 \mathrm{C}$ & 0.9800 \\
$\mathrm{O} 1-\mathrm{C} 1$ & $1.4708(19)$ & $\mathrm{C} 10-\mathrm{H} 10 \mathrm{~A}$ & 0.9800 \\
$\mathrm{~N} 1-\mathrm{C} 2$ & $1.405(2)$ & $\mathrm{C} 10-\mathrm{H} 10 \mathrm{~B}$ & 0.9800 \\
$\mathrm{~N} 1-\mathrm{C} 1$ & $1.447(2)$ & $\mathrm{C} 10-\mathrm{H} 10 \mathrm{C}$ & 0.9800 \\
$\mathrm{~N} 1-\mathrm{C} 9$ & $1.457(2)$ & $\mathrm{C} 11-\mathrm{H} 11 \mathrm{~A}$ & 0.9800 \\
$\mathrm{C} 1-\mathrm{C} 12$ & $1.496(2)$ & $\mathrm{C} 11-\mathrm{H} 11 \mathrm{~B}$ & 0.9800 \\
$\mathrm{C} 1-\mathrm{C} 8$ & $1.580(2)$ & $\mathrm{C} 11-\mathrm{H} 11 \mathrm{C}$ & 0.9800 \\
$\mathrm{C} 2-\mathrm{C} 3$ & $1.388(2)$ & $\mathrm{C} 12-\mathrm{C} 13$ & $1.330(3)$
\end{tabular}




\begin{tabular}{|c|c|c|c|}
\hline $\mathrm{C} 2-\mathrm{C} 7$ & $1.397(2)$ & $\mathrm{C} 12-\mathrm{H} 12$ & 0.9500 \\
\hline $\mathrm{C} 3-\mathrm{C} 4$ & $1.395(3)$ & $\mathrm{C} 13-\mathrm{C} 14$ & $1.452(2)$ \\
\hline $\mathrm{C} 3-\mathrm{H} 3$ & 0.9500 & $\mathrm{C} 13-\mathrm{H} 13$ & 0.9500 \\
\hline $\mathrm{C} 4-\mathrm{C} 5$ & $1.387(3)$ & $\mathrm{C} 14-\mathrm{C} 19$ & $1.397(2)$ \\
\hline $\mathrm{C} 4-\mathrm{H} 4$ & 0.9500 & $\mathrm{C} 14-\mathrm{C} 15$ & $1.398(2)$ \\
\hline $\mathrm{C} 5-\mathrm{C} 6$ & $1.398(3)$ & $\mathrm{C} 15-\mathrm{C} 16$ & $1.382(3)$ \\
\hline $\mathrm{C} 5-\mathrm{H} 5$ & 0.9500 & $\mathrm{C} 15-\mathrm{H} 15$ & 0.9500 \\
\hline $\mathrm{C} 6-\mathrm{C} 7$ & $1.381(2)$ & $\mathrm{C} 16-\mathrm{C} 17$ & $1.386(3)$ \\
\hline $\mathrm{C} 6-\mathrm{H} 6$ & 0.9500 & $\mathrm{C} 16-\mathrm{H} 16$ & 0.9500 \\
\hline $\mathrm{C} 7-\mathrm{C} 8$ & $1.511(2)$ & $\mathrm{C} 17-\mathrm{C} 18$ & $1.391(2)$ \\
\hline $\mathrm{C} 8-\mathrm{C} 11$ & $1.528(2)$ & C17-H17 & 0.9500 \\
\hline $\mathrm{C} 8-\mathrm{C} 10$ & $1.538(2)$ & $\mathrm{C} 18-\mathrm{C} 19$ & $1.387(2)$ \\
\hline C9-H9A & 0.9800 & $\mathrm{C} 18-\mathrm{H} 18$ & 0.9500 \\
\hline C9-H9B & 0.9800 & & \\
\hline $\mathrm{C} 19-\mathrm{O} 1-\mathrm{C} 1$ & $121.03(12)$ & $\mathrm{H} 9 \mathrm{~A}-\mathrm{C} 9-\mathrm{H} 9 \mathrm{C}$ & 109.5 \\
\hline $\mathrm{C} 2-\mathrm{N} 1-\mathrm{C} 1$ & $107.85(13)$ & $\mathrm{H} 9 \mathrm{~B}-\mathrm{C} 9-\mathrm{H} 9 \mathrm{C}$ & 109.5 \\
\hline $\mathrm{C} 2-\mathrm{N} 1-\mathrm{C} 9$ & $120.85(14)$ & $\mathrm{C} 8-\mathrm{C} 10-\mathrm{H} 10 \mathrm{~A}$ & 109.5 \\
\hline $\mathrm{C} 1-\mathrm{N} 1-\mathrm{C} 9$ & $119.66(14)$ & $\mathrm{C} 8-\mathrm{C} 10-\mathrm{H} 10 \mathrm{~B}$ & 109.5 \\
\hline $\mathrm{N} 1-\mathrm{C} 1-\mathrm{O} 1$ & $105.75(12)$ & $\mathrm{H} 10 \mathrm{~A}-\mathrm{C} 10-\mathrm{H} 10 \mathrm{~B}$ & 109.5 \\
\hline $\mathrm{N} 1-\mathrm{C} 1-\mathrm{C} 12$ & $112.92(14)$ & $\mathrm{C} 8-\mathrm{C} 10-\mathrm{H} 10 \mathrm{C}$ & 109.5 \\
\hline $\mathrm{O} 1-\mathrm{C} 1-\mathrm{C} 12$ & $111.35(13)$ & $\mathrm{H} 10 \mathrm{~A}-\mathrm{C} 10-\mathrm{H} 10 \mathrm{C}$ & 109.5 \\
\hline $\mathrm{N} 1-\mathrm{C} 1-\mathrm{C} 8$ & $102.85(13)$ & $\mathrm{H} 10 \mathrm{~B}-\mathrm{C} 10-\mathrm{H} 10 \mathrm{C}$ & 109.5 \\
\hline $\mathrm{O} 1-\mathrm{C} 1-\mathrm{C} 8$ & $108.57(12)$ & $\mathrm{C} 8-\mathrm{C} 11-\mathrm{H} 11 \mathrm{~A}$ & 109.5 \\
\hline $\mathrm{C} 12-\mathrm{C} 1-\mathrm{C} 8$ & $114.70(13)$ & $\mathrm{C} 8-\mathrm{C} 11-\mathrm{H} 11 \mathrm{~B}$ & 109.5 \\
\hline $\mathrm{C} 3-\mathrm{C} 2-\mathrm{C} 7$ & $121.35(16)$ & $\mathrm{H} 11 \mathrm{~A}-\mathrm{C} 11-\mathrm{H} 11 \mathrm{~B}$ & 109.5 \\
\hline $\mathrm{C} 3-\mathrm{C} 2-\mathrm{N} 1$ & $128.78(16)$ & $\mathrm{C} 8-\mathrm{C} 11-\mathrm{H} 11 \mathrm{C}$ & 109.5 \\
\hline $\mathrm{C} 7-\mathrm{C} 2-\mathrm{N} 1$ & $109.87(14)$ & $\mathrm{H} 11 \mathrm{~A}-\mathrm{C} 11-\mathrm{H} 11 \mathrm{C}$ & 109.5 \\
\hline $\mathrm{C} 2-\mathrm{C} 3-\mathrm{C} 4$ & $117.80(17)$ & $\mathrm{H} 11 \mathrm{~B}-\mathrm{C} 11-\mathrm{H} 11 \mathrm{C}$ & 109.5 \\
\hline $\mathrm{C} 2-\mathrm{C} 3-\mathrm{H} 3$ & 121.1 & $\mathrm{C} 13-\mathrm{C} 12-\mathrm{C} 1$ & $122.43(16)$ \\
\hline $\mathrm{C} 4-\mathrm{C} 3-\mathrm{H} 3$ & 121.1 & $\mathrm{C} 13-\mathrm{C} 12-\mathrm{H} 12$ & 118.8 \\
\hline $\mathrm{C} 5-\mathrm{C} 4-\mathrm{C} 3$ & $121.40(17)$ & $\mathrm{C} 1-\mathrm{C} 12-\mathrm{H} 12$ & 118.8 \\
\hline $\mathrm{C} 5-\mathrm{C} 4-\mathrm{H} 4$ & 119.3 & $\mathrm{C} 12-\mathrm{C} 13-\mathrm{C} 14$ & $121.19(16)$ \\
\hline $\mathrm{C} 3-\mathrm{C} 4-\mathrm{H} 4$ & 119.3 & $\mathrm{C} 12-\mathrm{C} 13-\mathrm{H} 13$ & 119.4 \\
\hline $\mathrm{C} 4-\mathrm{C} 5-\mathrm{C} 6$ & $120.06(17)$ & $\mathrm{C} 14-\mathrm{C} 13-\mathrm{H} 13$ & 119.4 \\
\hline $\mathrm{C} 4-\mathrm{C} 5-\mathrm{H} 5$ & 120.0 & $\mathrm{C} 19-\mathrm{C} 14-\mathrm{C} 15$ & $118.85(16)$ \\
\hline $\mathrm{C} 6-\mathrm{C} 5-\mathrm{H} 5$ & 120.0 & $\mathrm{C} 19-\mathrm{C} 14-\mathrm{C} 13$ & $117.40(15)$ \\
\hline $\mathrm{C} 7-\mathrm{C} 6-\mathrm{C} 5$ & $119.14(16)$ & $\mathrm{C} 15-\mathrm{C} 14-\mathrm{C} 13$ & $123.71(16)$ \\
\hline $\mathrm{C} 7-\mathrm{C} 6-\mathrm{H} 6$ & 120.4 & $\mathrm{C} 16-\mathrm{C} 15-\mathrm{C} 14$ & $120.83(17)$ \\
\hline $\mathrm{C} 5-\mathrm{C} 6-\mathrm{H} 6$ & 120.4 & $\mathrm{C} 16-\mathrm{C} 15-\mathrm{H} 15$ & 119.6 \\
\hline $\mathrm{C} 6-\mathrm{C} 7-\mathrm{C} 2$ & $120.22(16)$ & $\mathrm{C} 14-\mathrm{C} 15-\mathrm{H} 15$ & 119.6 \\
\hline $\mathrm{C} 6-\mathrm{C} 7-\mathrm{C} 8$ & $130.76(15)$ & $\mathrm{C} 15-\mathrm{C} 16-\mathrm{C} 17$ & $119.62(17)$ \\
\hline $\mathrm{C} 2-\mathrm{C} 7-\mathrm{C} 8$ & $108.96(14)$ & $\mathrm{C} 15-\mathrm{C} 16-\mathrm{H} 16$ & 120.2 \\
\hline $\mathrm{C} 7-\mathrm{C} 8-\mathrm{C} 11$ & $114.54(14)$ & $\mathrm{C} 17-\mathrm{C} 16-\mathrm{H} 16$ & 120.2 \\
\hline $\mathrm{C} 7-\mathrm{C} 8-\mathrm{C} 10$ & $109.56(13)$ & $\mathrm{C} 16-\mathrm{C} 17-\mathrm{C} 18$ & $120.55(17)$ \\
\hline $\mathrm{C} 11-\mathrm{C} 8-\mathrm{C} 10$ & $108.83(13)$ & $\mathrm{C} 16-\mathrm{C} 17-\mathrm{H} 17$ & 119.7 \\
\hline $\mathrm{C} 7-\mathrm{C} 8-\mathrm{C} 1$ & $100.34(12)$ & $\mathrm{C} 18-\mathrm{C} 17-\mathrm{H} 17$ & 119.7 \\
\hline $\mathrm{C} 11-\mathrm{C} 8-\mathrm{C} 1$ & $112.92(13)$ & $\mathrm{C} 19-\mathrm{C} 18-\mathrm{C} 17$ & $119.56(16)$ \\
\hline
\end{tabular}




\begin{tabular}{|c|c|c|c|}
\hline $\mathrm{C} 10-\mathrm{C} 8-\mathrm{C} 1$ & $110.41(13)$ & $\mathrm{C} 19-\mathrm{C} 18-\mathrm{H} 18$ & 120.2 \\
\hline $\mathrm{N} 1-\mathrm{C} 9-\mathrm{H} 9 \mathrm{~A}$ & 109.5 & $\mathrm{C} 17-\mathrm{C} 18-\mathrm{H} 18$ & 120.2 \\
\hline $\mathrm{N} 1-\mathrm{C} 9-\mathrm{H} 9 \mathrm{~B}$ & 109.5 & $\mathrm{O} 1-\mathrm{C} 19-\mathrm{C} 18$ & $117.61(14)$ \\
\hline $\mathrm{H} 9 \mathrm{~A}-\mathrm{C} 9-\mathrm{H} 9 \mathrm{~B}$ & 109.5 & $\mathrm{O} 1-\mathrm{C} 19-\mathrm{C} 14$ & $121.79(15)$ \\
\hline $\mathrm{N} 1-\mathrm{C} 9-\mathrm{H} 9 \mathrm{C}$ & 109.5 & $\mathrm{C} 18-\mathrm{C} 19-\mathrm{C} 14$ & $120.54(15)$ \\
\hline $\mathrm{C} 2-\mathrm{N} 1-\mathrm{C} 1-\mathrm{O} 1$ & $-82.59(15)$ & $\mathrm{C} 2-\mathrm{C} 7-\mathrm{C} 8-\mathrm{C} 1$ & $18.11(16)$ \\
\hline $\mathrm{C} 9-\mathrm{N} 1-\mathrm{C} 1-\mathrm{O} 1$ & $60.87(18)$ & $\mathrm{N} 1-\mathrm{C} 1-\mathrm{C} 8-\mathrm{C} 7$ & $-29.16(14)$ \\
\hline $\mathrm{C} 2-\mathrm{N} 1-\mathrm{C} 1-\mathrm{C} 12$ & $155.41(14)$ & $\mathrm{O} 1-\mathrm{C} 1-\mathrm{C} 8-\mathrm{C} 7$ & $82.58(14)$ \\
\hline $\mathrm{C} 9-\mathrm{N} 1-\mathrm{C} 1-\mathrm{C} 12$ & $-61.1(2)$ & $\mathrm{C} 12-\mathrm{C} 1-\mathrm{C} 8-\mathrm{C} 7$ & $-152.16(14)$ \\
\hline $\mathrm{C} 2-\mathrm{N} 1-\mathrm{C} 1-\mathrm{C} 8$ & $31.23(16)$ & $\mathrm{N} 1-\mathrm{C} 1-\mathrm{C} 8-\mathrm{C} 11$ & $-151.54(13)$ \\
\hline $\mathrm{C} 9-\mathrm{N} 1-\mathrm{C} 1-\mathrm{C} 8$ & $174.69(14)$ & $\mathrm{O} 1-\mathrm{C} 1-\mathrm{C} 8-\mathrm{C} 11$ & $-39.80(17)$ \\
\hline $\mathrm{C} 19-\mathrm{O} 1-\mathrm{C} 1-\mathrm{N} 1$ & $-148.68(13)$ & $\mathrm{C} 12-\mathrm{C} 1-\mathrm{C} 8-\mathrm{C} 11$ & $85.46(17)$ \\
\hline $\mathrm{C} 19-\mathrm{O} 1-\mathrm{C} 1-\mathrm{C} 12$ & $-25.67(19)$ & $\mathrm{N} 1-\mathrm{C} 1-\mathrm{C} 8-\mathrm{C} 10$ & $86.38(15)$ \\
\hline $\mathrm{C} 19-\mathrm{O} 1-\mathrm{C} 1-\mathrm{C} 8$ & $101.54(15)$ & $\mathrm{O} 1-\mathrm{C} 1-\mathrm{C} 8-\mathrm{C} 10$ & $-161.88(12)$ \\
\hline $\mathrm{C} 1-\mathrm{N} 1-\mathrm{C} 2-\mathrm{C} 3$ & $159.89(16)$ & $\mathrm{C} 12-\mathrm{C} 1-\mathrm{C} 8-\mathrm{C} 10$ & $-36.62(19)$ \\
\hline $\mathrm{C} 9-\mathrm{N} 1-\mathrm{C} 2-\mathrm{C} 3$ & $16.9(3)$ & $\mathrm{N} 1-\mathrm{C} 1-\mathrm{C} 12-\mathrm{C} 13$ & $139.09(17)$ \\
\hline $\mathrm{C} 1-\mathrm{N} 1-\mathrm{C} 2-\mathrm{C} 7$ & $-20.76(18)$ & $\mathrm{O} 1-\mathrm{C} 1-\mathrm{C} 12-\mathrm{C} 13$ & $20.3(2)$ \\
\hline $\mathrm{C} 9-\mathrm{N} 1-\mathrm{C} 2-\mathrm{C} 7$ & $-163.71(14)$ & $\mathrm{C} 8-\mathrm{C} 1-\mathrm{C} 12-\mathrm{C} 13$ & $-103.50(19)$ \\
\hline $\mathrm{C} 7-\mathrm{C} 2-\mathrm{C} 3-\mathrm{C} 4$ & $-1.8(2)$ & $\mathrm{C} 1-\mathrm{C} 12-\mathrm{C} 13-\mathrm{C} 14$ & $-5.3(3)$ \\
\hline $\mathrm{N} 1-\mathrm{C} 2-\mathrm{C} 3-\mathrm{C} 4$ & $177.50(16)$ & $\mathrm{C} 12-\mathrm{C} 13-\mathrm{C} 14-\mathrm{C} 19$ & $-6.3(2)$ \\
\hline $\mathrm{C} 2-\mathrm{C} 3-\mathrm{C} 4-\mathrm{C} 5$ & $0.5(3)$ & $\mathrm{C} 12-\mathrm{C} 13-\mathrm{C} 14-\mathrm{C} 15$ & $175.75(16)$ \\
\hline $\mathrm{C} 3-\mathrm{C} 4-\mathrm{C} 5-\mathrm{C} 6$ & $0.5(3)$ & $\mathrm{C} 19-\mathrm{C} 14-\mathrm{C} 15-\mathrm{C} 16$ & $0.5(2)$ \\
\hline $\mathrm{C} 4-\mathrm{C} 5-\mathrm{C} 6-\mathrm{C} 7$ & $-0.3(2)$ & $\mathrm{C} 13-\mathrm{C} 14-\mathrm{C} 15-\mathrm{C} 16$ & $178.41(15)$ \\
\hline $\mathrm{C} 5-\mathrm{C} 6-\mathrm{C} 7-\mathrm{C} 2$ & $-1.0(2)$ & $\mathrm{C} 14-\mathrm{C} 15-\mathrm{C} 16-\mathrm{C} 17$ & $-1.3(2)$ \\
\hline $\mathrm{C} 5-\mathrm{C} 6-\mathrm{C} 7-\mathrm{C} 8$ & $-177.85(15)$ & $\mathrm{C} 15-\mathrm{C} 16-\mathrm{C} 17-\mathrm{C} 18$ & $0.2(2)$ \\
\hline $\mathrm{C} 3-\mathrm{C} 2-\mathrm{C} 7-\mathrm{C} 6$ & $2.1(2)$ & $\mathrm{C} 16-\mathrm{C} 17-\mathrm{C} 18-\mathrm{C} 19$ & $1.8(2)$ \\
\hline $\mathrm{N} 1-\mathrm{C} 2-\mathrm{C} 7-\mathrm{C} 6$ & $-177.35(14)$ & $\mathrm{C} 1-\mathrm{O} 1-\mathrm{C} 19-\mathrm{C} 18$ & $-166.22(13)$ \\
\hline $\mathrm{C} 3-\mathrm{C} 2-\mathrm{C} 7-\mathrm{C} 8$ & $179.55(15)$ & $\mathrm{C} 1-\mathrm{O} 1-\mathrm{C} 19-\mathrm{C} 14$ & $16.5(2)$ \\
\hline $\mathrm{N} 1-\mathrm{C} 2-\mathrm{C} 7-\mathrm{C} 8$ & $0.14(18)$ & $\mathrm{C} 17-\mathrm{C} 18-\mathrm{C} 19-\mathrm{O} 1$ & $180.00(14)$ \\
\hline $\mathrm{C} 6-\mathrm{C} 7-\mathrm{C} 8-\mathrm{C} 11$ & $-43.5(2)$ & $\mathrm{C} 17-\mathrm{C} 18-\mathrm{C} 19-\mathrm{C} 14$ & $-2.7(2)$ \\
\hline $\mathrm{C} 2-\mathrm{C} 7-\mathrm{C} 8-\mathrm{C} 11$ & $139.34(14)$ & $\mathrm{C} 15-\mathrm{C} 14-\mathrm{C} 19-\mathrm{O} 1$ & $178.75(14)$ \\
\hline $\mathrm{C} 6-\mathrm{C} 7-\mathrm{C} 8-\mathrm{C} 10$ & $79.1(2)$ & $\mathrm{C} 13-\mathrm{C} 14-\mathrm{C} 19-\mathrm{O} 1$ & $0.7(2)$ \\
\hline $\mathrm{C} 2-\mathrm{C} 7-\mathrm{C} 8-\mathrm{C} 10$ & $-98.05(15)$ & $\mathrm{C} 15-\mathrm{C} 14-\mathrm{C} 19-\mathrm{C} 18$ & $1.5(2)$ \\
\hline $\mathrm{C} 6-\mathrm{C} 7-\mathrm{C} 8-\mathrm{C} 1$ & $-164.75(16)$ & $\mathrm{C} 13-\mathrm{C} 14-\mathrm{C} 19-\mathrm{C} 18$ & $-176.51(14)$ \\
\hline
\end{tabular}

(2) 1',3',3'-Trimethyl-4-[(E)-(1,3,3-trimethylindolin-2-ylidene) methyl]spiro[chroman-2,2'-indoline]

\section{Crystal data}

$\mathrm{C}_{31} \mathrm{H}_{34} \mathrm{~N}_{2} \mathrm{O}$

$M_{r}=450.60$

Monoclinic, $P 2_{1} / c$

$a=14.1774$ (11) $\AA$

$b=11.6019$ (9) $\AA$

$c=16.2847$ (17) $\AA$

$\beta=115.6129(12)^{\circ}$

$V=2415.4(4) \AA^{3}$

$Z=4$
$F(000)=968$

$D_{\mathrm{x}}=1.239 \mathrm{Mg} \mathrm{m}^{-3}$

Mo $K \alpha$ radiation, $\lambda=0.71073 \AA$

Cell parameters from 9967 reflections

$\theta=2.3-31.0^{\circ}$

$\mu=0.07 \mathrm{~mm}^{-1}$

$T=90 \mathrm{~K}$

Plate, colorless

$0.48 \times 0.26 \times 0.08 \mathrm{~mm}$ 


\section{Data collection}

Bruker DUO

diffractometer

Radiation source: fine focus sealed tube

Detector resolution: 8.3 pixels $\mathrm{mm}^{-1}$

$\omega$ scans

Absorption correction: multi-scan

(SADABS; Bruker, 2014)

$T_{\min }=0.713, T_{\max }=0.746$

\section{Refinement}

Refinement on $F^{2}$

Least-squares matrix: full

$R\left[F^{2}>2 \sigma\left(F^{2}\right)\right]=0.046$

$w R\left(F^{2}\right)=0.124$

$S=1.03$

7680 reflections

313 parameters

0 restraints

Primary atom site location: structure-invariant direct methods
39237 measured reflections

7680 independent reflections

6549 reflections with $I>2 \sigma(I)$

$R_{\text {int }}=0.026$

$\theta_{\text {max }}=31.0^{\circ}, \theta_{\min }=2.4^{\circ}$

$h=-20 \rightarrow 20$

$k=-16 \rightarrow 16$

$l=-23 \rightarrow 23$

Secondary atom site location: difference Fourier map

Hydrogen site location: inferred from

neighbouring sites

$\mathrm{H}$-atom parameters constrained

$w=1 /\left[\sigma^{2}\left(F_{\mathrm{o}}^{2}\right)+(0.0646 P)^{2}+0.9489 P\right]$

where $P=\left(F_{\mathrm{o}}{ }^{2}+2 F_{\mathrm{c}}{ }^{2}\right) / 3$

$(\Delta / \sigma)_{\max }<0.001$

$\Delta \rho_{\max }=0.61 \mathrm{e} \AA^{-3}$

$\Delta \rho_{\min }=-0.22$ e $\AA^{-3}$

Special details

Geometry. All esds (except the esd in the dihedral angle between two 1.s. planes) are estimated using the full covariance matrix. The cell esds are taken into account individually in the estimation of esds in distances, angles and torsion angles; correlations between esds in cell parameters are only used when they are defined by crystal symmetry. An approximate (isotropic) treatment of cell esds is used for estimating esds involving 1.s. planes.

Fractional atomic coordinates and isotropic or equivalent isotropic displacement parameters $\left(\AA^{2}\right)$

\begin{tabular}{lllll}
\hline & $x$ & $y$ & $z$ & $U_{\text {iso }} * / U_{\text {eq }}$ \\
\hline O1 & $0.34762(5)$ & $0.16535(6)$ & $0.32478(5)$ & $0.01634(15)$ \\
N1 & $0.40423(7)$ & $0.35186(7)$ & $0.37594(6)$ & $0.01547(16)$ \\
N2 & $-0.08953(7)$ & $0.35771(8)$ & $-0.01968(6)$ & $0.01639(17)$ \\
C1 & $0.31133(7)$ & $0.27916(8)$ & $0.33767(7)$ & $0.01376(17)$ \\
C2 & $0.45179(8)$ & $0.33748(8)$ & $0.47069(7)$ & $0.01494(18)$ \\
C3 & $0.55233(8)$ & $0.36930(9)$ & $0.53229(7)$ & $0.01845(19)$ \\
H3 & 0.5998 & 0.4030 & 0.5122 & $0.022^{*}$ \\
C4 & $0.58087(9)$ & $0.34990(10)$ & $0.62471(7)$ & $0.0212(2)$ \\
H4 & 0.6489 & 0.3712 & 0.6682 & $0.025^{*}$ \\
C5 & $0.51211(9)$ & $0.30028(10)$ & $0.65452(8)$ & $0.0231(2)$ \\
H5 & 0.5335 & 0.2875 & 0.7177 & $0.028^{*}$ \\
C6 & $0.41093(9)$ & $0.26899(10)$ & $0.59145(7)$ & $0.0211(2)$ \\
H6 & 0.3633 & 0.2355 & 0.6114 & $0.025^{*}$ \\
C7 & $0.38174(8)$ & $0.28778(9)$ & $0.49969(7)$ & $0.01572(18)$ \\
C8 & $0.27724(7)$ & $0.27236(8)$ & $0.41788(7)$ & $0.01457(17)$ \\
C9 & $0.47220(8)$ & $0.36495(10)$ & $0.33042(7)$ & $0.0195(2)$ \\
H9A & 0.5114 & 0.4372 & 0.3498 & $0.029^{*}$ \\
H9B & 0.4297 & 0.3664 & 0.2643 & $0.029 *$ \\
H9C & 0.5212 & 0.3001 & 0.3464 & $0.029 *$ \\
C10 & $0.20944(8)$ & $0.37710(10)$ & $0.41676(8)$ & $0.0203(2)$
\end{tabular}




\begin{tabular}{|c|c|c|c|c|}
\hline $\mathrm{H} 10 \mathrm{~A}$ & 0.2004 & 0.3784 & 0.4731 & $0.031 *$ \\
\hline H10B & 0.1408 & 0.3711 & 0.3644 & $0.031 *$ \\
\hline $\mathrm{H} 10 \mathrm{C}$ & 0.2440 & 0.4482 & 0.4120 & $0.031 *$ \\
\hline $\mathrm{C} 11$ & $0.22236(8)$ & $0.16035(10)$ & $0.42081(7)$ & $0.0203(2)$ \\
\hline H11A & 0.2120 & 0.1582 & 0.4765 & $0.030 *$ \\
\hline H11B & 0.2655 & 0.0946 & 0.4202 & $0.030 *$ \\
\hline $\mathrm{H} 11 \mathrm{C}$ & 0.1544 & 0.1563 & 0.3676 & $0.030 *$ \\
\hline $\mathrm{C} 12$ & $0.23105(8)$ & $0.32528(9)$ & $0.24650(7)$ & $0.01534(18)$ \\
\hline $\mathrm{H} 12 \mathrm{~A}$ & 0.2665 & 0.3408 & 0.2070 & $0.018 *$ \\
\hline H12B & 0.2034 & 0.3994 & 0.2569 & $0.018^{*}$ \\
\hline $\mathrm{C} 13$ & $0.13889(7)$ & $0.24303(8)$ & $0.19642(7)$ & $0.01457(17)$ \\
\hline H13 & 0.0967 & 0.2367 & 0.2320 & $0.017 *$ \\
\hline $\mathrm{C} 14$ & $0.18259(7)$ & $0.12496(8)$ & $0.19179(6)$ & $0.01406(17)$ \\
\hline $\mathrm{C} 15$ & $0.12593(8)$ & $0.04410(9)$ & $0.12446(7)$ & 0.01773 (19) \\
\hline H15 & 0.0575 & 0.0634 & 0.0805 & $0.021 *$ \\
\hline $\mathrm{C} 16$ & $0.16694(8)$ & $-0.06372(9)$ & $0.12009(7)$ & $0.0192(2)$ \\
\hline H16 & 0.1272 & -0.1169 & 0.0736 & $0.023 *$ \\
\hline $\mathrm{C} 17$ & $0.26725(8)$ & $-0.09253(9)$ & $0.18494(8)$ & $0.0198(2)$ \\
\hline H17 & 0.2960 & -0.1658 & 0.1827 & $0.024 *$ \\
\hline C18 & $0.32501(8)$ & $-0.01440(9)$ & $0.25269(7)$ & 0.01735 (19) \\
\hline H18 & 0.3930 & -0.0344 & 0.2969 & $0.021 *$ \\
\hline C19 & $0.28304(7)$ & $0.09388(8)$ & $0.25583(7)$ & $0.01425(17)$ \\
\hline $\mathrm{C} 20$ & $0.06994(8)$ & $0.28955(9)$ & $0.10287(7)$ & $0.01557(18)$ \\
\hline $\mathrm{H} 20$ & 0.1026 & 0.3022 & 0.0635 & $0.019 *$ \\
\hline $\mathrm{C} 21$ & $-0.03249(7)$ & $0.31510(8)$ & $0.06905(6)$ & $0.01364(17)$ \\
\hline $\mathrm{C} 22$ & $-0.19342(8)$ & $0.37872(8)$ & $-0.03783(7)$ & $0.01475(18)$ \\
\hline $\mathrm{C} 23$ & $-0.27463(8)$ & $0.42123(9)$ & $-0.11683(7)$ & $0.01866(19)$ \\
\hline $\mathrm{H} 23$ & -0.2638 & 0.4406 & -0.1688 & $0.022 *$ \\
\hline $\mathrm{C} 24$ & $-0.37283(8)$ & $0.43437(10)$ & $-0.11672(8)$ & $0.0213(2)$ \\
\hline $\mathrm{H} 24$ & -0.4295 & 0.4630 & -0.1698 & $0.026^{*}$ \\
\hline $\mathrm{C} 25$ & $-0.38953(8)$ & $0.40669(10)$ & $-0.04102(8)$ & $0.0211(2)$ \\
\hline $\mathrm{H} 25$ & -0.4569 & 0.4165 & -0.0427 & $0.025^{*}$ \\
\hline $\mathrm{C} 26$ & $-0.30669(8)$ & $0.36425(9)$ & $0.03782(7)$ & 0.01789 (19) \\
\hline $\mathrm{H} 26$ & -0.3173 & 0.3453 & 0.0899 & $0.021 *$ \\
\hline $\mathrm{C} 27$ & $-0.20940(7)$ & $0.35039(8)$ & $0.03879(7)$ & 0.01449 (18) \\
\hline $\mathrm{C} 28$ & $-0.10793(7)$ & $0.30639(8)$ & $0.11424(6)$ & $0.01381(17)$ \\
\hline $\mathrm{C} 29$ & $-0.07525(8)$ & $0.38417(10)$ & $0.19859(7)$ & $0.0196(2)$ \\
\hline $\mathrm{H} 29 \mathrm{~A}$ & -0.0588 & 0.4614 & 0.1842 & $0.029 *$ \\
\hline H29B & -0.0134 & 0.3515 & 0.2488 & $0.029 *$ \\
\hline $\mathrm{H} 29 \mathrm{C}$ & -0.1327 & 0.3891 & 0.2166 & $0.029 *$ \\
\hline $\mathrm{C} 30$ & $-0.12197(9)$ & $0.18061(9)$ & $0.13746(8)$ & $0.0211(2)$ \\
\hline $\mathrm{H} 30 \mathrm{~A}$ & -0.1753 & 0.1774 & 0.1606 & $0.032 *$ \\
\hline H30B & -0.0555 & 0.1515 & 0.1840 & $0.032 *$ \\
\hline $\mathrm{H} 30 \mathrm{C}$ & -0.1441 & 0.1331 & 0.0825 & $0.032 *$ \\
\hline $\mathrm{C} 31$ & $-0.04513(9)$ & $0.37235(10)$ & $-0.08373(7)$ & $0.0209(2)$ \\
\hline H31A & -0.0985 & 0.4030 & -0.1411 & $0.031 *$ \\
\hline H31B & -0.0205 & 0.2977 & -0.0950 & $0.031 *$ \\
\hline $\mathrm{H} 31 \mathrm{C}$ & 0.0138 & 0.4263 & -0.0586 & $0.031 *$ \\
\hline
\end{tabular}


Atomic displacement parameters $\left(\AA^{2}\right)$

\begin{tabular}{|c|c|c|c|c|c|c|}
\hline & $U^{11}$ & $U^{22}$ & $U^{33}$ & $U^{12}$ & $U^{13}$ & $U^{23}$ \\
\hline $\mathrm{O} 1$ & 0.0140 & $0.0146(3)$ & $0.0178(3)$ & $0.0019(2)$ & 0.0043 & -0.0028 \\
\hline N1 & $0.0150(4)$ & $0.0173(4)$ & $0.0146(4)$ & -0.0025 & 0.0069 & -0.0006 \\
\hline $\mathrm{N} 2$ & $0.0156(4)$ & $0.0212(4)$ & $0.0129(4)$ & 0.0024 & 0.0068 & 0.0041 \\
\hline $\mathrm{C} 1$ & $0.0142(4)$ & $0.0130(4)$ & $0.0143(4)$ & 0.0015 & $0.0064(3)$ & $0.0002(3)$ \\
\hline $\mathrm{C} 2$ & $0.0160(4)$ & $0.0139(4)$ & $0.0147(4)$ & $0.0016(3)$ & 0.0065 & $-0.0006(3)$ \\
\hline $\mathrm{C} 3$ & $0.0164(4)$ & $0.0180(4)$ & $0.0202(5)$ & -0.0006 & $0.0072(4)$ & $-0.0026(4)$ \\
\hline $\mathrm{C} 4$ & $0.0187(5)$ & $0.0217(5)$ & $0.0187(5)$ & 0.0013 & $0.0041(4)$ & $-0.0034(4)$ \\
\hline $\mathrm{C} 5$ & $0.0246(5)$ & $0.0263(5)$ & $0.0149(4)$ & $0.0016(4)$ & $0.0054(4)$ & 0.0008 (4) \\
\hline C6 & $0.0226(5)$ & $0.0247(5)$ & $0.0168(5)$ & $0.0005(4)$ & $0.0091(4)$ & $0.0026(4)$ \\
\hline $\mathrm{C} 7$ & $0.0161(4)$ & $0.0156(4)$ & $0.0154(4)$ & 0.0023 & 0.0067 & 0.0013 \\
\hline $\mathrm{C} 8$ & $0.0149(4)$ & $0.0159(4)$ & $0.0141(4)$ & 0.0011 & 0.0074 & $0.0014(3)$ \\
\hline C9 & $0.0186(4)$ & $0.0232(5)$ & $0.0200(5)$ & -0.0021 & $0.0115(4)$ & $0.0004(4)$ \\
\hline $\mathrm{C} 10$ & $0.0187(4)$ & $0.0227(5)$ & $0.0201(5)$ & $0.0041(4)$ & 0.0088 & -0.0019 (4) \\
\hline $\mathrm{C} 11$ & $0.0212(5)$ & $0.0220(5)$ & $0.0191(5)$ & $-0.0041(4)$ & $0.0100(4)$ & $0.0017(4)$ \\
\hline $\mathrm{C} 12$ & 0.0160 & $0.0150(4)$ & $0.0140(4)$ & 0.0010 & 0.0055 & 0.0010 \\
\hline C13 & $0.0142(4)$ & $0.0156(4)$ & 0.0139 (4) & 0.0021 & 0.0061 & 0.0014 \\
\hline $\mathrm{C} 14$ & 0.0140 & $0.0154(4)$ & $0.0135(4)$ & 0.0009 & 0.0066 & 0.0005 \\
\hline $\mathrm{C} 15$ & $0.0174(4)$ & $0.0191(5)$ & $0.0153(4)$ & -0.0009 (3) & 0.0057 & 0.0000 \\
\hline $\mathrm{C} 16$ & $0.0231(5)$ & $0.0174(4)$ & $0.0172(4)$ & -0.0030 & 0.0088 & -0.0038 \\
\hline $\mathrm{C} 17$ & $0.0218(5)$ & $0.0169(4)$ & $0.0230(5)$ & 0.0008 & $0.0118(4)$ & -0.0025 \\
\hline $\mathrm{C} 18$ & $0.0155(4)$ & $0.0172(4)$ & $0.0204(5)$ & 0.0021 & $0.0087(4)$ & -0.0004 \\
\hline C19 & $0.0136(4)$ & $0.0150(4)$ & $0.0153(4)$ & 0.0001 & 0.0074 & $-0.0006(3)$ \\
\hline $\mathrm{C} 20$ & $0.0157(4)$ & $0.0181(4)$ & $0.0136(4)$ & 0.0018 & 0.0069 & $0.0017(3)$ \\
\hline $\mathrm{C} 21$ & $0.0164(4)$ & $0.0132(4)$ & $0.0117(4)$ & $0.0002(3)$ & 0.0064 & 0.0005 \\
\hline $\mathrm{C} 22$ & $0.0154(4)$ & $0.0132(4)$ & $0.0141(4)$ & $-0.0007(3)$ & 0.0050 & $-0.0006(3)$ \\
\hline $\mathrm{C} 23$ & $0.0199(5)$ & $0.0180(5)$ & $0.0144(4)$ & $0.0000(4)$ & 0.0039 (4) & $0.0014(3)$ \\
\hline $\mathrm{C} 24$ & $0.0173(4)$ & $0.0200(5)$ & $0.0200(5)$ & 0.0008 & 0.0018 (4) & 0.0007 (4) \\
\hline $\mathrm{C} 25$ & $0.0140(4)$ & $0.0212(5)$ & $0.0241(5)$ & $-0.0007(4)$ & $0.0046(4)$ & -0.0007 (4) \\
\hline $\mathrm{C} 26$ & $0.0152(4)$ & 0.0188 & $0.0190(5)$ & -0.0024 & 0.0067 (4) & -0.0009 (4) \\
\hline $\mathrm{C} 27$ & $0.0141(4)$ & $0.0133(4)$ & $0.0148(4)$ & -0.0013 & $0.0051(3)$ & $-0.0004(3)$ \\
\hline $\mathrm{C} 28$ & $0.0144(4)$ & $0.0148(4)$ & $0.0130(4)$ & 0.0005 & $0.0066(3)$ & $0.0013(3)$ \\
\hline $\mathrm{C} 29$ & $0.0169(4)$ & $0.0265(5)$ & $0.0143(4)$ & $0.0026(4)$ & 0.0057 (4) & $-0.0025(4)$ \\
\hline $\mathrm{C} 30$ & $0.0210(5)$ & $0.0184(5)$ & $0.0255(5)$ & 0.0000 & $0.0116(4)$ & $0.0067(4)$ \\
\hline C31 & $0.0222(5)$ & $0.0276(5)$ & $0.0158(4)$ & $0.0015(4)$ & $0.0111(4)$ & $0.0042(4)$ \\
\hline
\end{tabular}

Geometric parameters $\left(\AA,{ }^{\circ}\right)$

\begin{tabular}{llll}
\hline $\mathrm{O} 1-\mathrm{C} 19$ & $1.3776(12)$ & $\mathrm{C} 13-\mathrm{H} 13$ & 1.0000 \\
$\mathrm{O} 1-\mathrm{C} 1$ & $1.4648(12)$ & $\mathrm{C} 14-\mathrm{C} 19$ & $1.4005(13)$ \\
$\mathrm{N} 1-\mathrm{C} 2$ & $1.4013(13)$ & $\mathrm{C} 14-\mathrm{C} 15$ & $1.4022(14)$ \\
$\mathrm{N} 1-\mathrm{C} 9$ & $1.4556(13)$ & $\mathrm{C} 15-\mathrm{C} 16$ & 0.9500 \\
$\mathrm{~N} 1-\mathrm{C} 1$ & $1.4577(13)$ & $\mathrm{C} 15-\mathrm{H} 15$ & $1.3967(15)$ \\
$\mathrm{N} 2-\mathrm{C} 22$ & $1.3928(13)$ & $\mathrm{C} 16-\mathrm{C} 17$ & 0.9500 \\
$\mathrm{~N} 2-\mathrm{C} 21$ & $1.4056(12)$ & $\mathrm{C} 16-\mathrm{H} 16$ & $1.3887(15)$ \\
$\mathrm{N} 2-\mathrm{C} 31$ & $1.4425(13)$ & $\mathrm{C} 17-\mathrm{C} 18$ &
\end{tabular}




\begin{tabular}{|c|c|c|c|}
\hline $\mathrm{C} 1-\mathrm{C} 12$ & $1.5247(13)$ & $\mathrm{C} 17-\mathrm{H} 17$ & 0.9500 \\
\hline $\mathrm{C} 1-\mathrm{C} 8$ & $1.5785(14)$ & $\mathrm{C} 18-\mathrm{C} 19$ & $1.4004(14)$ \\
\hline $\mathrm{C} 2-\mathrm{C} 3$ & $1.3921(14)$ & $\mathrm{C} 18-\mathrm{H} 18$ & 0.9500 \\
\hline $\mathrm{C} 2-\mathrm{C} 7$ & $1.3956(14)$ & $\mathrm{C} 20-\mathrm{C} 21$ & $1.3448(13)$ \\
\hline $\mathrm{C} 3-\mathrm{C} 4$ & $1.3975(15)$ & $\mathrm{C} 20-\mathrm{H} 20$ & 0.9500 \\
\hline $\mathrm{C} 3-\mathrm{H} 3$ & 0.9500 & $\mathrm{C} 21-\mathrm{C} 28$ & 1.5415 (13) \\
\hline $\mathrm{C} 4-\mathrm{C} 5$ & $1.3881(17)$ & $\mathrm{C} 22-\mathrm{C} 23$ & $1.3941(13)$ \\
\hline $\mathrm{C} 4-\mathrm{H} 4$ & 0.9500 & $\mathrm{C} 22-\mathrm{C} 27$ & $1.4002(14)$ \\
\hline $\mathrm{C} 5-\mathrm{C} 6$ & $1.4037(16)$ & $\mathrm{C} 23-\mathrm{C} 24$ & $1.4013(15)$ \\
\hline $\mathrm{C} 5-\mathrm{H} 5$ & 0.9500 & $\mathrm{C} 23-\mathrm{H} 23$ & 0.9500 \\
\hline $\mathrm{C} 6-\mathrm{C} 7$ & $1.3845(14)$ & $\mathrm{C} 24-\mathrm{C} 25$ & $1.3896(16)$ \\
\hline $\mathrm{C} 6-\mathrm{H} 6$ & 0.9500 & $\mathrm{C} 24-\mathrm{H} 24$ & 0.9500 \\
\hline $\mathrm{C} 7-\mathrm{C} 8$ & $1.5150(14)$ & $\mathrm{C} 25-\mathrm{C} 26$ & $1.4023(14)$ \\
\hline $\mathrm{C} 8-\mathrm{C} 11$ & $1.5260(14)$ & $\mathrm{C} 25-\mathrm{H} 25$ & 0.9500 \\
\hline $\mathrm{C} 8-\mathrm{C} 10$ & $1.5446(14)$ & $\mathrm{C} 26-\mathrm{C} 27$ & $1.3820(14)$ \\
\hline C9-H9A & 0.9800 & $\mathrm{C} 26-\mathrm{H} 26$ & 0.9500 \\
\hline C9-H9B & 0.9800 & $\mathrm{C} 27-\mathrm{C} 28$ & $1.5204(13)$ \\
\hline $\mathrm{C} 9-\mathrm{H} 9 \mathrm{C}$ & 0.9800 & $\mathrm{C} 28-\mathrm{C} 29$ & $1.5381(14)$ \\
\hline $\mathrm{C} 10-\mathrm{H} 10 \mathrm{~A}$ & 0.9800 & $\mathrm{C} 28-\mathrm{C} 30$ & $1.5418(14)$ \\
\hline $\mathrm{C} 10-\mathrm{H} 10 \mathrm{~B}$ & 0.9800 & $\mathrm{C} 29-\mathrm{H} 29 \mathrm{~A}$ & 0.9800 \\
\hline $\mathrm{C} 10-\mathrm{H} 10 \mathrm{C}$ & 0.9800 & $\mathrm{C} 29-\mathrm{H} 29 \mathrm{~B}$ & 0.9800 \\
\hline $\mathrm{C} 11-\mathrm{H} 11 \mathrm{~A}$ & 0.9800 & $\mathrm{C} 29-\mathrm{H} 29 \mathrm{C}$ & 0.9800 \\
\hline $\mathrm{C} 11-\mathrm{H} 11 \mathrm{~B}$ & 0.9800 & $\mathrm{C} 30-\mathrm{H} 30 \mathrm{~A}$ & 0.9800 \\
\hline $\mathrm{C} 11-\mathrm{H} 11 \mathrm{C}$ & 0.9800 & $\mathrm{C} 30-\mathrm{H} 30 \mathrm{~B}$ & 0.9800 \\
\hline $\mathrm{C} 12-\mathrm{C} 13$ & $1.5367(14)$ & $\mathrm{C} 30-\mathrm{H} 30 \mathrm{C}$ & 0.9800 \\
\hline $\mathrm{C} 12-\mathrm{H} 12 \mathrm{~A}$ & 0.9900 & $\mathrm{C} 31-\mathrm{H} 31 \mathrm{~A}$ & 0.9800 \\
\hline $\mathrm{C} 12-\mathrm{H} 12 \mathrm{~B}$ & 0.9900 & $\mathrm{C} 31-\mathrm{H} 31 \mathrm{~B}$ & 0.9800 \\
\hline $\mathrm{C} 13-\mathrm{C} 20$ & $1.5100(13)$ & $\mathrm{C} 31-\mathrm{H} 31 \mathrm{C}$ & 0.9800 \\
\hline $\mathrm{C} 13-\mathrm{C} 14$ & $1.5186(14)$ & & \\
\hline $\mathrm{C} 19-\mathrm{O} 1-\mathrm{C} 1$ & $120.56(7)$ & $\mathrm{C} 19-\mathrm{C} 14-\mathrm{C} 15$ & $117.67(9)$ \\
\hline $\mathrm{C} 2-\mathrm{N} 1-\mathrm{C} 9$ & $117.63(8)$ & $\mathrm{C} 19-\mathrm{C} 14-\mathrm{C} 13$ & $120.04(8)$ \\
\hline $\mathrm{C} 2-\mathrm{N} 1-\mathrm{C} 1$ & $108.51(8)$ & $\mathrm{C} 15-\mathrm{C} 14-\mathrm{C} 13$ & $122.29(9)$ \\
\hline $\mathrm{C} 9-\mathrm{N} 1-\mathrm{C} 1$ & $121.16(8)$ & $\mathrm{C} 16-\mathrm{C} 15-\mathrm{C} 14$ & $121.98(9)$ \\
\hline $\mathrm{C} 22-\mathrm{N} 2-\mathrm{C} 21$ & $111.43(8)$ & $\mathrm{C} 16-\mathrm{C} 15-\mathrm{H} 15$ & 119.0 \\
\hline $\mathrm{C} 22-\mathrm{N} 2-\mathrm{C} 31$ & $125.31(8)$ & $\mathrm{C} 14-\mathrm{C} 15-\mathrm{H} 15$ & 119.0 \\
\hline $\mathrm{C} 21-\mathrm{N} 2-\mathrm{C} 31$ & $123.21(8)$ & $\mathrm{C} 15-\mathrm{C} 16-\mathrm{C} 17$ & $119.14(9)$ \\
\hline $\mathrm{N} 1-\mathrm{C} 1-\mathrm{O} 1$ & $105.93(7)$ & $\mathrm{C} 15-\mathrm{C} 16-\mathrm{H} 16$ & 120.4 \\
\hline $\mathrm{N} 1-\mathrm{C} 1-\mathrm{C} 12$ & $111.68(8)$ & $\mathrm{C} 17-\mathrm{C} 16-\mathrm{H} 16$ & 120.4 \\
\hline $\mathrm{O} 1-\mathrm{C} 1-\mathrm{C} 12$ & $109.72(8)$ & $\mathrm{C} 18-\mathrm{C} 17-\mathrm{C} 16$ & $120.16(10)$ \\
\hline $\mathrm{N} 1-\mathrm{C} 1-\mathrm{C} 8$ & $102.62(8)$ & $\mathrm{C} 18-\mathrm{C} 17-\mathrm{H} 17$ & 119.9 \\
\hline $\mathrm{O} 1-\mathrm{C} 1-\mathrm{C} 8$ & $109.01(7)$ & $\mathrm{C} 16-\mathrm{C} 17-\mathrm{H} 17$ & 119.9 \\
\hline $\mathrm{C} 12-\mathrm{C} 1-\mathrm{C} 8$ & $117.17(8)$ & $\mathrm{C} 17-\mathrm{C} 18-\mathrm{C} 19$ & $120.04(9)$ \\
\hline $\mathrm{C} 3-\mathrm{C} 2-\mathrm{C} 7$ & $121.49(9)$ & $\mathrm{C} 17-\mathrm{C} 18-\mathrm{H} 18$ & 120.0 \\
\hline $\mathrm{C} 3-\mathrm{C} 2-\mathrm{N} 1$ & $128.12(9)$ & $\mathrm{C} 19-\mathrm{C} 18-\mathrm{H} 18$ & 120.0 \\
\hline $\mathrm{C} 7-\mathrm{C} 2-\mathrm{N} 1$ & $110.35(8)$ & $\mathrm{O} 1-\mathrm{C} 19-\mathrm{C} 18$ & $115.25(8)$ \\
\hline $\mathrm{C} 2-\mathrm{C} 3-\mathrm{C} 4$ & $117.58(10)$ & $\mathrm{O} 1-\mathrm{C} 19-\mathrm{C} 14$ & $123.75(9)$ \\
\hline $\mathrm{C} 2-\mathrm{C} 3-\mathrm{H} 3$ & 121.2 & $\mathrm{C} 18-\mathrm{C} 19-\mathrm{C} 14$ & $121.00(9)$ \\
\hline
\end{tabular}




\begin{tabular}{|c|c|c|c|}
\hline $\mathrm{C} 4-\mathrm{C} 3-\mathrm{H} 3$ & 121.2 & $\mathrm{C} 21-\mathrm{C} 20-\mathrm{C} 13$ & $127.27(9)$ \\
\hline $\mathrm{C} 5-\mathrm{C} 4-\mathrm{C} 3$ & $121.55(10)$ & $\mathrm{C} 21-\mathrm{C} 20-\mathrm{H} 20$ & 116.4 \\
\hline $\mathrm{C} 5-\mathrm{C} 4-\mathrm{H} 4$ & 119.2 & $\mathrm{C} 13-\mathrm{C} 20-\mathrm{H} 20$ & 116.4 \\
\hline $\mathrm{C} 3-\mathrm{C} 4-\mathrm{H} 4$ & 119.2 & $\mathrm{C} 20-\mathrm{C} 21-\mathrm{N} 2$ & $122.55(9)$ \\
\hline $\mathrm{C} 4-\mathrm{C} 5-\mathrm{C} 6$ & $120.10(10)$ & $\mathrm{C} 20-\mathrm{C} 21-\mathrm{C} 28$ & $129.73(9)$ \\
\hline $\mathrm{C} 4-\mathrm{C} 5-\mathrm{H} 5$ & 120.0 & $\mathrm{~N} 2-\mathrm{C} 21-\mathrm{C} 28$ & $107.71(8)$ \\
\hline $\mathrm{C} 6-\mathrm{C} 5-\mathrm{H} 5$ & 120.0 & $\mathrm{~N} 2-\mathrm{C} 22-\mathrm{C} 23$ & $129.31(9)$ \\
\hline $\mathrm{C} 7-\mathrm{C} 6-\mathrm{C} 5$ & $118.89(10)$ & $\mathrm{N} 2-\mathrm{C} 22-\mathrm{C} 27$ & $109.48(8)$ \\
\hline $\mathrm{C} 7-\mathrm{C} 6-\mathrm{H} 6$ & 120.6 & $\mathrm{C} 23-\mathrm{C} 22-\mathrm{C} 27$ & $121.21(9)$ \\
\hline $\mathrm{C} 5-\mathrm{C} 6-\mathrm{H} 6$ & 120.6 & $\mathrm{C} 22-\mathrm{C} 23-\mathrm{C} 24$ & $117.51(10)$ \\
\hline $\mathrm{C} 6-\mathrm{C} 7-\mathrm{C} 2$ & $120.39(9)$ & $\mathrm{C} 22-\mathrm{C} 23-\mathrm{H} 23$ & 121.2 \\
\hline $\mathrm{C} 6-\mathrm{C} 7-\mathrm{C} 8$ & $130.77(9)$ & $\mathrm{C} 24-\mathrm{C} 23-\mathrm{H} 23$ & 121.2 \\
\hline $\mathrm{C} 2-\mathrm{C} 7-\mathrm{C} 8$ & $108.64(8)$ & $\mathrm{C} 25-\mathrm{C} 24-\mathrm{C} 23$ & $121.77(10)$ \\
\hline $\mathrm{C} 7-\mathrm{C} 8-\mathrm{C} 11$ & $113.02(8)$ & $\mathrm{C} 25-\mathrm{C} 24-\mathrm{H} 24$ & 119.1 \\
\hline $\mathrm{C} 7-\mathrm{C} 8-\mathrm{C} 10$ & $106.52(8)$ & $\mathrm{C} 23-\mathrm{C} 24-\mathrm{H} 24$ & 119.1 \\
\hline $\mathrm{C} 11-\mathrm{C} 8-\mathrm{C} 10$ & $110.32(8)$ & $\mathrm{C} 24-\mathrm{C} 25-\mathrm{C} 26$ & $119.79(10)$ \\
\hline $\mathrm{C} 7-\mathrm{C} 8-\mathrm{C} 1$ & $100.90(8)$ & $\mathrm{C} 24-\mathrm{C} 25-\mathrm{H} 25$ & 120.1 \\
\hline $\mathrm{C} 11-\mathrm{C} 8-\mathrm{C} 1$ & $114.27(8)$ & $\mathrm{C} 26-\mathrm{C} 25-\mathrm{H} 25$ & 120.1 \\
\hline $\mathrm{C} 10-\mathrm{C} 8-\mathrm{C} 1$ & $111.26(8)$ & $\mathrm{C} 27-\mathrm{C} 26-\mathrm{C} 25$ & $119.21(10)$ \\
\hline $\mathrm{N} 1-\mathrm{C} 9-\mathrm{H} 9 \mathrm{~A}$ & 109.5 & $\mathrm{C} 27-\mathrm{C} 26-\mathrm{H} 26$ & 120.4 \\
\hline $\mathrm{N} 1-\mathrm{C} 9-\mathrm{H} 9 \mathrm{~B}$ & 109.5 & $\mathrm{C} 25-\mathrm{C} 26-\mathrm{H} 26$ & 120.4 \\
\hline $\mathrm{H} 9 \mathrm{~A}-\mathrm{C} 9-\mathrm{H} 9 \mathrm{~B}$ & 109.5 & $\mathrm{C} 26-\mathrm{C} 27-\mathrm{C} 22$ & $120.51(9)$ \\
\hline $\mathrm{N} 1-\mathrm{C} 9-\mathrm{H} 9 \mathrm{C}$ & 109.5 & $\mathrm{C} 26-\mathrm{C} 27-\mathrm{C} 28$ & $129.74(9)$ \\
\hline $\mathrm{H} 9 \mathrm{~A}-\mathrm{C} 9-\mathrm{H} 9 \mathrm{C}$ & 109.5 & $\mathrm{C} 22-\mathrm{C} 27-\mathrm{C} 28$ & $109.75(8)$ \\
\hline $\mathrm{H} 9 \mathrm{~B}-\mathrm{C} 9-\mathrm{H} 9 \mathrm{C}$ & 109.5 & $\mathrm{C} 27-\mathrm{C} 28-\mathrm{C} 29$ & $109.83(8)$ \\
\hline $\mathrm{C} 8-\mathrm{C} 10-\mathrm{H} 10 \mathrm{~A}$ & 109.5 & $\mathrm{C} 27-\mathrm{C} 28-\mathrm{C} 21$ & $101.63(8)$ \\
\hline $\mathrm{C} 8-\mathrm{C} 10-\mathrm{H} 10 \mathrm{~B}$ & 109.5 & $\mathrm{C} 29-\mathrm{C} 28-\mathrm{C} 21$ & $112.62(8)$ \\
\hline $\mathrm{H} 10 \mathrm{~A}-\mathrm{C} 10-\mathrm{H} 10 \mathrm{~B}$ & 109.5 & $\mathrm{C} 27-\mathrm{C} 28-\mathrm{C} 30$ & $109.79(8)$ \\
\hline $\mathrm{C} 8-\mathrm{C} 10-\mathrm{H} 10 \mathrm{C}$ & 109.5 & $\mathrm{C} 29-\mathrm{C} 28-\mathrm{C} 30$ & $110.93(8)$ \\
\hline $\mathrm{H} 10 \mathrm{~A}-\mathrm{C} 10-\mathrm{H} 10 \mathrm{C}$ & 109.5 & $\mathrm{C} 21-\mathrm{C} 28-\mathrm{C} 30$ & $111.65(8)$ \\
\hline $\mathrm{H} 10 \mathrm{~B}-\mathrm{C} 10-\mathrm{H} 10 \mathrm{C}$ & 109.5 & $\mathrm{C} 28-\mathrm{C} 29-\mathrm{H} 29 \mathrm{~A}$ & 109.5 \\
\hline $\mathrm{C} 8-\mathrm{C} 11-\mathrm{H} 11 \mathrm{~A}$ & 109.5 & $\mathrm{C} 28-\mathrm{C} 29-\mathrm{H} 29 \mathrm{~B}$ & 109.5 \\
\hline $\mathrm{C} 8-\mathrm{C} 11-\mathrm{H} 11 \mathrm{~B}$ & 109.5 & $\mathrm{H} 29 \mathrm{~A}-\mathrm{C} 29-\mathrm{H} 29 \mathrm{~B}$ & 109.5 \\
\hline $\mathrm{H} 11 \mathrm{~A}-\mathrm{C} 11-\mathrm{H} 11 \mathrm{~B}$ & 109.5 & $\mathrm{C} 28-\mathrm{C} 29-\mathrm{H} 29 \mathrm{C}$ & 109.5 \\
\hline $\mathrm{C} 8-\mathrm{C} 11-\mathrm{H} 11 \mathrm{C}$ & 109.5 & $\mathrm{H} 29 \mathrm{~A}-\mathrm{C} 29-\mathrm{H} 29 \mathrm{C}$ & 109.5 \\
\hline $\mathrm{H} 11 \mathrm{~A}-\mathrm{C} 11-\mathrm{H} 11 \mathrm{C}$ & 109.5 & $\mathrm{H} 29 \mathrm{~B}-\mathrm{C} 29-\mathrm{H} 29 \mathrm{C}$ & 109.5 \\
\hline $\mathrm{H} 11 \mathrm{~B}-\mathrm{C} 11-\mathrm{H} 11 \mathrm{C}$ & 109.5 & $\mathrm{C} 28-\mathrm{C} 30-\mathrm{H} 30 \mathrm{~A}$ & 109.5 \\
\hline $\mathrm{C} 1-\mathrm{C} 12-\mathrm{C} 13$ & $113.85(8)$ & $\mathrm{C} 28-\mathrm{C} 30-\mathrm{H} 30 \mathrm{~B}$ & 109.5 \\
\hline $\mathrm{C} 1-\mathrm{C} 12-\mathrm{H} 12 \mathrm{~A}$ & 108.8 & $\mathrm{H} 30 \mathrm{~A}-\mathrm{C} 30-\mathrm{H} 30 \mathrm{~B}$ & 109.5 \\
\hline $\mathrm{C} 13-\mathrm{C} 12-\mathrm{H} 12 \mathrm{~A}$ & 108.8 & $\mathrm{C} 28-\mathrm{C} 30-\mathrm{H} 30 \mathrm{C}$ & 109.5 \\
\hline $\mathrm{C} 1-\mathrm{C} 12-\mathrm{H} 12 \mathrm{~B}$ & 108.8 & $\mathrm{H} 30 \mathrm{~A}-\mathrm{C} 30-\mathrm{H} 30 \mathrm{C}$ & 109.5 \\
\hline $\mathrm{C} 13-\mathrm{C} 12-\mathrm{H} 12 \mathrm{~B}$ & 108.8 & $\mathrm{H} 30 \mathrm{~B}-\mathrm{C} 30-\mathrm{H} 30 \mathrm{C}$ & 109.5 \\
\hline $\mathrm{H} 12 \mathrm{~A}-\mathrm{C} 12-\mathrm{H} 12 \mathrm{~B}$ & 107.7 & $\mathrm{~N} 2-\mathrm{C} 31-\mathrm{H} 31 \mathrm{~A}$ & 109.5 \\
\hline $\mathrm{C} 20-\mathrm{C} 13-\mathrm{C} 14$ & $111.87(8)$ & $\mathrm{N} 2-\mathrm{C} 31-\mathrm{H} 31 \mathrm{~B}$ & 109.5 \\
\hline $\mathrm{C} 20-\mathrm{C} 13-\mathrm{C} 12$ & $110.16(8)$ & $\mathrm{H} 31 \mathrm{~A}-\mathrm{C} 31-\mathrm{H} 31 \mathrm{~B}$ & 109.5 \\
\hline $\mathrm{C} 14-\mathrm{C} 13-\mathrm{C} 12$ & $108.36(8)$ & $\mathrm{N} 2-\mathrm{C} 31-\mathrm{H} 31 \mathrm{C}$ & 109.5 \\
\hline $\mathrm{C} 20-\mathrm{C} 13-\mathrm{H} 13$ & 108.8 & $\mathrm{H} 31 \mathrm{~A}-\mathrm{C} 31-\mathrm{H} 31 \mathrm{C}$ & 109.5 \\
\hline $\mathrm{C} 14-\mathrm{C} 13-\mathrm{H} 13$ & 108.8 & $\mathrm{H} 31 \mathrm{~B}-\mathrm{C} 31-\mathrm{H} 31 \mathrm{C}$ & 109.5 \\
\hline
\end{tabular}




\begin{tabular}{|c|c|c|c|}
\hline $\mathrm{C} 12-\mathrm{C} 13-\mathrm{H} 13$ & 108.8 & & \\
\hline $\mathrm{C} 2-\mathrm{N} 1-\mathrm{C} 1-\mathrm{O} 1$ & $-85.70(9)$ & $\mathrm{C} 19-\mathrm{C} 14-\mathrm{C} 15-\mathrm{C} 16$ & $-0.24(15)$ \\
\hline $\mathrm{C} 9-\mathrm{N} 1-\mathrm{C} 1-\mathrm{O} 1$ & $55.02(11)$ & $\mathrm{C} 13-\mathrm{C} 14-\mathrm{C} 15-\mathrm{C} 16$ & $179.36(9)$ \\
\hline $\mathrm{C} 2-\mathrm{N} 1-\mathrm{C} 1-\mathrm{C} 12$ & $154.89(8)$ & $\mathrm{C} 14-\mathrm{C} 15-\mathrm{C} 16-\mathrm{C} 17$ & $0.36(16)$ \\
\hline $\mathrm{C} 9-\mathrm{N} 1-\mathrm{C} 1-\mathrm{C} 12$ & $-64.39(11)$ & $\mathrm{C} 15-\mathrm{C} 16-\mathrm{C} 17-\mathrm{C} 18$ & $-0.04(16)$ \\
\hline $\mathrm{C} 2-\mathrm{N} 1-\mathrm{C} 1-\mathrm{C} 8$ & $28.56(10)$ & $\mathrm{C} 16-\mathrm{C} 17-\mathrm{C} 18-\mathrm{C} 19$ & $-0.40(16)$ \\
\hline $\mathrm{C} 9-\mathrm{N} 1-\mathrm{C} 1-\mathrm{C} 8$ & $169.28(8)$ & $\mathrm{C} 1-\mathrm{O} 1-\mathrm{C} 19-\mathrm{C} 18$ & $-177.53(8)$ \\
\hline $\mathrm{C} 19-\mathrm{O} 1-\mathrm{C} 1-\mathrm{N} 1$ & $-149.92(8)$ & $\mathrm{C} 1-\mathrm{O} 1-\mathrm{C} 19-\mathrm{C} 14$ & $3.06(14)$ \\
\hline $\mathrm{C} 19-\mathrm{O} 1-\mathrm{C} 1-\mathrm{C} 12$ & $-29.24(11)$ & $\mathrm{C} 17-\mathrm{C} 18-\mathrm{C} 19-\mathrm{O} 1$ & $-178.90(9)$ \\
\hline $\mathrm{C} 19-\mathrm{O} 1-\mathrm{C} 1-\mathrm{C} 8$ & $100.29(10)$ & $\mathrm{C} 17-\mathrm{C} 18-\mathrm{C} 19-\mathrm{C} 14$ & $0.53(15)$ \\
\hline $\mathrm{C} 9-\mathrm{N} 1-\mathrm{C} 2-\mathrm{C} 3$ & $22.49(15)$ & $\mathrm{C} 15-\mathrm{C} 14-\mathrm{C} 19-\mathrm{O} 1$ & $179.16(9)$ \\
\hline $\mathrm{C} 1-\mathrm{N} 1-\mathrm{C} 2-\mathrm{C} 3$ & $164.79(10)$ & $\mathrm{C} 13-\mathrm{C} 14-\mathrm{C} 19-\mathrm{O} 1$ & $-0.44(14)$ \\
\hline $\mathrm{C} 9-\mathrm{N} 1-\mathrm{C} 2-\mathrm{C} 7$ & $-159.66(9)$ & $\mathrm{C} 15-\mathrm{C} 14-\mathrm{C} 19-\mathrm{C} 18$ & $-0.21(14)$ \\
\hline $\mathrm{C} 1-\mathrm{N} 1-\mathrm{C} 2-\mathrm{C} 7$ & $-17.36(11)$ & $\mathrm{C} 13-\mathrm{C} 14-\mathrm{C} 19-\mathrm{C} 18$ & $-179.82(9)$ \\
\hline $\mathrm{C} 7-\mathrm{C} 2-\mathrm{C} 3-\mathrm{C} 4$ & $-0.12(15)$ & $\mathrm{C} 14-\mathrm{C} 13-\mathrm{C} 20-\mathrm{C} 21$ & $117.80(11)$ \\
\hline $\mathrm{N} 1-\mathrm{C} 2-\mathrm{C} 3-\mathrm{C} 4$ & $177.51(10)$ & $\mathrm{C} 12-\mathrm{C} 13-\mathrm{C} 20-\mathrm{C} 21$ & $-121.61(11)$ \\
\hline $\mathrm{C} 2-\mathrm{C} 3-\mathrm{C} 4-\mathrm{C} 5$ & $0.29(16)$ & $\mathrm{C} 13-\mathrm{C} 20-\mathrm{C} 21-\mathrm{N} 2$ & $-179.70(9)$ \\
\hline $\mathrm{C} 3-\mathrm{C} 4-\mathrm{C} 5-\mathrm{C} 6$ & $-0.46(17)$ & $\mathrm{C} 13-\mathrm{C} 20-\mathrm{C} 21-\mathrm{C} 28$ & $0.79(18)$ \\
\hline $\mathrm{C} 4-\mathrm{C} 5-\mathrm{C} 6-\mathrm{C} 7$ & $0.44(17)$ & $\mathrm{C} 22-\mathrm{N} 2-\mathrm{C} 21-\mathrm{C} 20$ & $-179.21(9)$ \\
\hline $\mathrm{C} 5-\mathrm{C} 6-\mathrm{C} 7-\mathrm{C} 2$ & $-0.28(16)$ & $\mathrm{C} 31-\mathrm{N} 2-\mathrm{C} 21-\mathrm{C} 20$ & $3.22(16)$ \\
\hline $\mathrm{C} 5-\mathrm{C} 6-\mathrm{C} 7-\mathrm{C} 8$ & $-174.41(10)$ & $\mathrm{C} 22-\mathrm{N} 2-\mathrm{C} 21-\mathrm{C} 28$ & $0.39(11)$ \\
\hline $\mathrm{C} 3-\mathrm{C} 2-\mathrm{C} 7-\mathrm{C} 6$ & $0.12(15)$ & $\mathrm{C} 31-\mathrm{N} 2-\mathrm{C} 21-\mathrm{C} 28$ & $-177.17(9)$ \\
\hline $\mathrm{N} 1-\mathrm{C} 2-\mathrm{C} 7-\mathrm{C} 6$ & $-177.89(9)$ & $\mathrm{C} 21-\mathrm{N} 2-\mathrm{C} 22-\mathrm{C} 23$ & $179.71(10)$ \\
\hline $\mathrm{C} 3-\mathrm{C} 2-\mathrm{C} 7-\mathrm{C} 8$ & $175.44(9)$ & $\mathrm{C} 31-\mathrm{N} 2-\mathrm{C} 22-\mathrm{C} 23$ & $-2.79(17)$ \\
\hline $\mathrm{N} 1-\mathrm{C} 2-\mathrm{C} 7-\mathrm{C} 8$ & $-2.58(11)$ & $\mathrm{C} 21-\mathrm{N} 2-\mathrm{C} 22-\mathrm{C} 27$ & $-0.10(12)$ \\
\hline $\mathrm{C} 6-\mathrm{C} 7-\mathrm{C} 8-\mathrm{C} 11$ & $-43.62(15)$ & $\mathrm{C} 31-\mathrm{N} 2-\mathrm{C} 22-\mathrm{C} 27$ & $177.40(10)$ \\
\hline $\mathrm{C} 2-\mathrm{C} 7-\mathrm{C} 8-\mathrm{C} 11$ & $141.72(9)$ & $\mathrm{N} 2-\mathrm{C} 22-\mathrm{C} 23-\mathrm{C} 24$ & $-179.88(10)$ \\
\hline $\mathrm{C} 6-\mathrm{C} 7-\mathrm{C} 8-\mathrm{C} 10$ & $77.68(13)$ & $\mathrm{C} 27-\mathrm{C} 22-\mathrm{C} 23-\mathrm{C} 24$ & $-0.09(15)$ \\
\hline $\mathrm{C} 2-\mathrm{C} 7-\mathrm{C} 8-\mathrm{C} 10$ & $-96.98(10)$ & $\mathrm{C} 22-\mathrm{C} 23-\mathrm{C} 24-\mathrm{C} 25$ & $0.19(16)$ \\
\hline $\mathrm{C} 6-\mathrm{C} 7-\mathrm{C} 8-\mathrm{C} 1$ & $-166.08(11)$ & $\mathrm{C} 23-\mathrm{C} 24-\mathrm{C} 25-\mathrm{C} 26$ & $-0.08(17)$ \\
\hline $\mathrm{C} 2-\mathrm{C} 7-\mathrm{C} 8-\mathrm{C} 1$ & $19.26(10)$ & $\mathrm{C} 24-\mathrm{C} 25-\mathrm{C} 26-\mathrm{C} 27$ & $-0.14(16)$ \\
\hline $\mathrm{N} 1-\mathrm{C} 1-\mathrm{C} 8-\mathrm{C} 7$ & $-28.15(9)$ & $\mathrm{C} 25-\mathrm{C} 26-\mathrm{C} 27-\mathrm{C} 22$ & $0.23(15)$ \\
\hline $\mathrm{O} 1-\mathrm{C} 1-\mathrm{C} 8-\mathrm{C} 7$ & $83.84(9)$ & $\mathrm{C} 25-\mathrm{C} 26-\mathrm{C} 27-\mathrm{C} 28$ & $-179.83(10)$ \\
\hline $\mathrm{C} 12-\mathrm{C} 1-\mathrm{C} 8-\mathrm{C} 7$ & $-150.86(8)$ & $\mathrm{N} 2-\mathrm{C} 22-\mathrm{C} 27-\mathrm{C} 26$ & $179.71(9)$ \\
\hline $\mathrm{N} 1-\mathrm{C} 1-\mathrm{C} 8-\mathrm{C} 11$ & $-149.73(8)$ & $\mathrm{C} 23-\mathrm{C} 22-\mathrm{C} 27-\mathrm{C} 26$ & $-0.12(15)$ \\
\hline $\mathrm{O} 1-\mathrm{C} 1-\mathrm{C} 8-\mathrm{C} 11$ & $-37.73(11)$ & $\mathrm{N} 2-\mathrm{C} 22-\mathrm{C} 27-\mathrm{C} 28$ & $-0.24(11)$ \\
\hline $\mathrm{C} 12-\mathrm{C} 1-\mathrm{C} 8-\mathrm{C} 11$ & $87.57(10)$ & $\mathrm{C} 23-\mathrm{C} 22-\mathrm{C} 27-\mathrm{C} 28$ & $179.93(9)$ \\
\hline $\mathrm{N} 1-\mathrm{C} 1-\mathrm{C} 8-\mathrm{C} 10$ & $84.53(9)$ & $\mathrm{C} 26-\mathrm{C} 27-\mathrm{C} 28-\mathrm{C} 29$ & $-60.05(13)$ \\
\hline $\mathrm{O} 1-\mathrm{C} 1-\mathrm{C} 8-\mathrm{C} 10$ & $-163.48(8)$ & $\mathrm{C} 22-\mathrm{C} 27-\mathrm{C} 28-\mathrm{C} 29$ & $119.89(9)$ \\
\hline $\mathrm{C} 12-\mathrm{C} 1-\mathrm{C} 8-\mathrm{C} 10$ & $-38.18(11)$ & $\mathrm{C} 26-\mathrm{C} 27-\mathrm{C} 28-\mathrm{C} 21$ & $-179.50(10)$ \\
\hline $\mathrm{N} 1-\mathrm{C} 1-\mathrm{C} 12-\mathrm{C} 13$ & $171.51(8)$ & $\mathrm{C} 22-\mathrm{C} 27-\mathrm{C} 28-\mathrm{C} 21$ & $0.44(10)$ \\
\hline $\mathrm{O} 1-\mathrm{C} 1-\mathrm{C} 12-\mathrm{C} 13$ & $54.37(10)$ & $\mathrm{C} 26-\mathrm{C} 27-\mathrm{C} 28-\mathrm{C} 30$ & $62.18(13)$ \\
\hline $\mathrm{C} 8-\mathrm{C} 1-\mathrm{C} 12-\mathrm{C} 13$ & $-70.57(11)$ & $\mathrm{C} 22-\mathrm{C} 27-\mathrm{C} 28-\mathrm{C} 30$ & $-117.88(9)$ \\
\hline $\mathrm{C} 1-\mathrm{C} 12-\mathrm{C} 13-\mathrm{C} 20$ & $-173.83(8)$ & $\mathrm{C} 20-\mathrm{C} 21-\mathrm{C} 28-\mathrm{C} 27$ & $179.07(10)$ \\
\hline $\mathrm{C} 1-\mathrm{C} 12-\mathrm{C} 13-\mathrm{C} 14$ & $-51.16(11)$ & $\mathrm{N} 2-\mathrm{C} 21-\mathrm{C} 28-\mathrm{C} 27$ & $-0.49(10)$ \\
\hline $\mathrm{C} 20-\mathrm{C} 13-\mathrm{C} 14-\mathrm{C} 19$ & $145.65(9)$ & $\mathrm{C} 20-\mathrm{C} 21-\mathrm{C} 28-\mathrm{C} 29$ & $61.62(14)$ \\
\hline $\mathrm{C} 12-\mathrm{C} 13-\mathrm{C} 14-\mathrm{C} 19$ & $24.02(12)$ & $\mathrm{N} 2-\mathrm{C} 21-\mathrm{C} 28-\mathrm{C} 29$ & $-117.94(9)$ \\
\hline
\end{tabular}


$\mathrm{C} 20-\mathrm{C} 13-\mathrm{C} 14-\mathrm{C} 15-33.94(13)$

$\mathrm{C} 12-\mathrm{C} 13-\mathrm{C} 14-\mathrm{C} 15 \quad-155.57$ (9)
$\mathrm{C} 20-\mathrm{C} 21-\mathrm{C} 28-\mathrm{C} 30$

$\mathrm{N} 2-\mathrm{C} 21-\mathrm{C} 28-\mathrm{C} 30$
$-63.95(14)$

$116.48(9)$ 